\title{
Activation of LH GABAergic inputs counteracts fasting-induced changes in tVTA/RMTG
}

neurons.

Nathan Godfrey ${ }^{1}$, Min Qiao $^{1}$, and Stephanie L. Borgland ${ }^{1,2}$

${ }^{1}$ University of Calgary

Department of Physiology and Pharmacology

Calgary, Alberta

T2N 4N1

${ }^{2}$ To whom correspondence should be addressed:

s.borgland@ucalgary.ca

Running title: Acute fasting on LH GABA inputs to the tVTA/RMTg.

Key words: ventral tegmental area, fasting, dopamine, inhibitory synaptic transmission, tVTA, RMTg, GABA, motivational state. 


\section{Key Points:}

- While dopamine neuronal activity changes with motivational state, it is unknown if fasting influences tVTA/RMTg GABAergic neurons, a major inhibitory input to VTA dopamine neurons.

- In unfasted mice, there were sex differences in inhibitory synaptic transmission onto tVTA/RMTg GABAergic neurons.

- Activation of LH GABAergic neurons decreases firing of tVTA/RMTg GABAergic neurons through a monosynaptic input.

- An acute fast decreased the excitability of tVTA/RMTg GABAergic neurons.

- An acute fast decreases inhibitory synaptic transmission of the LH GABA input to tVTA/RMTg GABAergic neurons in both male and female mice.

\section{Abstract:}

Dopamine neurons in the ventral tegmental area (VTA) are strongly innervated by GABAergic neurons in the 'tail of the VTA' (tVTA), also known as the rostralmedial tegmental nucleus (RMTg). Disinhibition of dopamine neurons through firing of the GABAergic neurons projecting from the lateral hypothalamus (LH) leads to reward seeking and consumption through dopamine release in the nucleus accumbens. VTA dopamine neurons respond to changes in motivational state, yet less is known of whether tVTA/RMTg GABAergic neurons or the LH GABAergic neurons that project to them are also affected by changes in motivational state, such as fasting. An acute $16 \mathrm{~h}$ overnight fast decreased the excitability of tVTA/RMTg GABAergic neurons of male and female mice. In addition, fasting decreased synaptic strength at LH GABA to tVTA/RMTg GABAergic synapses, indicated by reduced amplitude of optically evoked currents, decreased readily releasable pool (RRP) size and replenishment. Optical stimulation of LH GABA terminals suppressed evoked action potentials of tVTA/RMTg GABAergic neurons in unfasted mice, but this effect decreased following fasting in both males and females. Furthermore, during fasting, LH GABA inputs to tVTA/RMTg neurons maintained 
functional connectivity during depolarization, as depolarization block was reduced following fasting. Taken together, inhibitory synaptic transmission from LH GABA inputs onto tVTA/RMTg GABAergic neurons decreases following fasting, however ability to functionally inhibit tVTA/RMTg GABAergic neurons is preserved, allowing for possible disinhibition of dopamine neurons and subsequent foraging.

\section{Introduction}

Motivated behaviors arise during physiological need. The ventral tegmental area (VTA) dopamine neurons play a critical role in encoding the salience of cues in the environment that predict the quality and availability of rewards and dopamine release in the nucleus accumbens (NAc) can motivate animals to seek out these rewards (Salamone et al., 2003; Wise, 2006;

Berridge, 2009). Dopamine neurons alter their firing activity and output in a state-dependent manner. For example, negative sodium balance increases phasic dopamine release in the NAC, whereas dopamine concentration was decreased when rats were sodium replete, suggesting that dopamine neurons track fluid balance in a state-dependent manner (Cone et al., 2016; Fortin \& Roitman, 2018). Similarly, VTA dopamine neurons respond to a motivational state induced by food deprivation. During an acute fast, there is a sex dependent increase in excitatory synaptic transmission onto dopamine neurons (Godfrey \& Borgland, 2020) as well as increased somatodendritic dopamine release measured by its effects on D2 receptor mediated inhibitory post synaptic currents (IPSCs) (Roseberry, 2015). Optogenetic activation of VTA dopamine neurons increases food retrieval in fasted animals, but prior acute exposure to high 
fat diet strongly suppresses this effect (Mazzone et al., 2020). Taken together, VTA dopamine neuronal activity changes with motivational state.

The VTA is composed of approximately $60-70 \%$ dopaminergic neurons, $25-30 \%$ GABAergic neurons and 2-5\% glutamatergic neurons (Margolis et al., 2006, 2012; Nair-Roberts et al., 2008; Chieng et al., 2011; Ungless \& Grace, 2012). The caudal region or the 'tail of the VTA' (tVTA) also known as the rostralmedial tegmental nucleus (RMTg) is composed of almost exclusively GABAergic neurons (Jhou et al., 2009). In brain atlases, the tVTA/RMTg is not differentiated from the VTA itself and thus non-projecting GABAergic neurons within the caudal region of the VTA are likely part of this structure. Dopamine neurons are the principal target of tVTA/RMTg neurons (Balcita-Pedicino et al., 2011). As such, these GABAergic neurons exert a significant inhibitory drive onto VTA dopaminergic neurons (Barrot et al., 2012). Optogenetic stimulation of VTA GABA neurons disrupts licking for sucrose solution in fasted mice (Van Zessen et al., 2012). Furthermore, a variety of aversive stimuli activate tVTA/RMTg neurons (Sánchez-Catalán et al., 2017; Li et al., 2019c). Given that hunger induced by acute fasting is an aversive motivational state (Betley et al., 2015), it is unknown if GABAergic neurons of the tVTA/RMTg are also sensitive to state-dependent changes in activity from fasting.

GABAergic neurons from the VTA or VVTA/RMTg receive a strong inhibitory input from the lateral hypothalamus (LH) (Nieh et al., 2015). Activation of LH GABAergic terminals in the VTA disinhibits VTA dopamine neurons and increases dopamine release in the NAc (Stuber \& Wise, 2016). Electrical or optogenetic stimulation of LH GABAergic neurons elicits robust feeding behaviour (Nieh et al., 2015; Stuber \& Wise, 2016). Some reports have indicated that activation of the LH GABAergic to VTA GABAergic projection elicits feeding behaviour through 
disinhibition of dopamine neurons (Nieh et al., 2015, 2016), while others suggest that activation of LH GABAergic neurons mediates feeding behaviour through an en passant projection through the VTA terminating near the locus coeruleus (Marino et al., 2020). Furthermore, while it was proposed that feeding evoked by stimulation of GABAergic LH to VTA projections was due to the rewarding properties of food rather than the aversive drive state associated with hunger, it was never specifically tested whether this input was sensitive to an acute fast (Nieh et al., 2016). Here, we tested whether caudal tVTA/RMTg GABAergic neurons were sensitive to an acute fast and how synaptic transmission of LH GABA to tVTA/RMTg GABAergic neuronal projection was altered by this motivationally relevant stimulus.

\section{Methods}

Land acknowledgement:

This work was conceived and performed at The University of Calgary, located on the traditional territories of the people of the Treaty 7 region in Southern Alberta, which includes the Blackfoot Confederacy (including the Siksika, Piikuni, Kainai First Nations), the Tsuut'ina, and the Stoney Nakoda (including the Chiniki, Bearspaw, and Wesley First Nations). The City of Calgary is also home to Metis Nation of Alberta, Region 3.

\section{Subjects}

All experiments and procedures were in accordance with the ethical guidelines established by the Canadian Council for Animal Care and were approved by the University of Calgary Animal 
Care Committee (protocol no. AC17-0034) and are consistent with the Journal's policies for the ethical treatment of animals (Grundy, 2015).

Male and female mice 2-4 months old were housed in groups of two to five in same sex cages and were maintained on a $12 \mathrm{~h}$ light-dark schedule (lights on at $08.00 \mathrm{~h}$, zeitgeber time (ZT) 0 and were given chow and water ad libitum. Mice were fed chow (5062 from Pico-Vac lab diet, Lab Supply, Fort Worth Tx), which is composed of (\% of total kcal) 23\% protein, 22\% fat (ether extract), and $55 \%$ carbohydrate. The total caloric density of this diet was $4.60 \mathrm{kcal} \mathrm{g}^{-1}$. Experiments were performed during the animal's light cycle. Male and female VGAT ${ }^{c r e} T d-$ Tomato mice, used to identify GABA neurons, were generated by crossing VGAT ${ }^{\text {cre }}$ (Slc32a1tm2(cre)Lowl/J) obtained from Jackson Laboratory (strain: 016962) with Rosa-td Tomato mice (B6.Cg-Gt[ROSA]26 Sortm9[CAG-tdTomato]Hze/J [Ai9]). All mice were bred locally in the Clara Christie Centre for Mouse Genomics. Mice were placed in a stereotaxic surgery frame (Kopf; Tujunga, CA) and channelrhodopsin (ChR2) (AAV2-EF1a-DIO-hChR2(H134)-EYFP; Neurophotonics, Centre de Recherche CERVO, Quebec City, QC, Canada) was bilaterally infused into the lateral hypothalamus (LH) with the following coordinates: ML: +/- $1.000 \mathrm{~mm}, \mathrm{AP}:-0.800$ $\mathrm{mm}$, DV: $-5.300 \mathrm{~mm}$. Mice were returned to home cages for 6 to 7 weeks for full virus expression before use in experiments. Mice were initially weighed and then isolated $10 \mathrm{~h}$ into their light phase (ZT10), and single-housed overnight for a total of $16 \mathrm{~h}$. During this time, food was removed from the cages of the fasted group and chow was given ad libitum to the control group. Water was given ad libitum to both groups. Water intake and body weight were recorded for fasted and control groups and food consumption per body weight was recorded in 
the control group after the $16 \mathrm{~h}$ fast (ZT2). Electrophysiology experiments were performed on mice at ZT2.

\section{Electrophysiology}

All electrophysiological recordings were performed in slice preparations from adult (2-4 months old) VGAT ${ }^{\text {cre }} \mathrm{Td}$-Tomato mice. In these mice, a red fluorescent marker is expressed exclusively in GABA neurons, permitting immediate identification of GABAergic neurons in the tVTA/RMTg for electrophysiological recordings. We recorded fluorescently identified GABAergic neurons from the caudal/medial VTA located medial to the medial lemniscus (ML). Mice were deeply anaesthetized with isoflurane and transcardially perfused with an ice-cold N-methyl-Dglucamine (NMDG) solution containing (in mM): $93 \mathrm{NMDG}, 2.5 \mathrm{KCl}, 1.2 \mathrm{NaH}_{2} \mathrm{PO}_{4} .2 \mathrm{H}_{2} \mathrm{O}, 30$ $\mathrm{NaHCO}_{3}, 20$ Hepes, 25 D-glucose, 5 sodium ascorbate, 3 sodium pyruvate, 2 thiourea, 10 $\mathrm{MgSO}_{4} .7 \mathrm{H}_{2} \mathrm{O}, 0.5 \mathrm{CaCl}_{2} .2 \mathrm{H}_{2} \mathrm{O}$ and saturated with $95 \% \mathrm{O}_{2}-5 \% \mathrm{CO}_{2}$. Mice were then decapitated, and the brain extracted. Horizontal midbrain sections $(250 \mu \mathrm{m})$ containing the VTA were cut on a vibrating-blade microtome (Leica, Nussloch, Germany). Slices were then incubated in NMDG solution $\left(32^{\circ} \mathrm{C}\right)$ and saturated with $95 \% \mathrm{O}_{2}-5 \% \mathrm{CO}_{2}$ for $10 \mathrm{~min}$. Following this, the slices were transferred to artificial cerebrospinal fluid (aCSF) containing (in mM): $126 \mathrm{NaCl}, 1.6 \mathrm{KCl}, 1.1$ $\mathrm{NaH}_{2} \mathrm{PO}_{4}, 1.4 \mathrm{MgCl}_{2}, 2.4 \mathrm{CaCl}_{2}, 26 \mathrm{NaHCO}_{3}, 11$ glucose, $\left(32^{\circ} \mathrm{C}\right)$ and saturated with $95 \% \mathrm{O}_{2}-5 \%$ $\mathrm{CO}_{2}$. Slices were incubated for a minimum of $45 \mathrm{~min}$ before being transferred to a recording chamber and superfused $\left(2 \mathrm{~mL} \mathrm{~min}^{-1}\right)$ with aCSF $\left(32-34^{\circ} \mathrm{C}\right)$ and saturated with $95 \% \mathrm{O}_{2}-5 \% \mathrm{CO}_{2}$. Neurons were visualized on an upright microscope using 'Dodt-type' gradient contrast infrared optics and whole-cell recordings were made using a MultiClamp 700B amplifier (Molecular 
Devices, San Jose, CA, USA). The recorded signal was collected at a sampling rate of $20 \mathrm{kHz}$ and a $2 \mathrm{kHz}$ Bessel filter was applied to the data during collection. For optically evoked currents, a 3 $\mathrm{ms}, 3.5 \mathrm{~mW}$, pulse of $470 \mathrm{~nm}$ was provided by an LED light source through a $\times 40 / 0.80$ water immersible Olympus microscope objective. Recorded neurons were positioned so that the soma was at the centre of the objective. All electrophysiology experiments were done in the presence of DNQX $(10 \mu \mathrm{M})$.

Voltage clamp recordings. For all voltage clamp recordings, recording electrodes (2-5 $\mathrm{M} \Omega$ ) were filled with Cesium Chloride internal solution containing (in mM): $140 \mathrm{CsCl}, 10 \mathrm{HEPES}, 0.2 \mathrm{EGTA}$, $1 \mathrm{MgCl}_{2}, 2 \mathrm{MgATP}, 0.3 \mathrm{NaGTP}, 5 \mathrm{QX}-314-\mathrm{Cl}$, and $0.2 \%$ Biocytin. The liquid junction potential was $3.5 \mathrm{mV}$, but not corrected for.

Validation of ChR2 expression and function. To validate and confirm the selective expression and function of ChR2 in GABA neurons of the $\mathrm{LH}$, train stimulations were evoked at $5 \mathrm{~Hz}, 10 \mathrm{~Hz}$, and $20 \mathrm{~Hz}$ recording from LH GABA neurons or tVTA/RMTg GABA neurons. Example traces are averages of 3 sweeps. A 1 second, continuous, stimulation was also optically evoked in both LH GABA neurons and tVTA/RMTg GABA neurons. Furthermore, optically evoked currents were recorded before and after the bath application of picrotoxin $(100 \mu \mathrm{M})$. The two minutes, 12 sweeps, prior to picrotoxin application were compared to the two minutes, 12 sweeps, after the effect of picrotoxin was seen to take full effect. Finally, optically evoked currents were recorded in the presence of TTX $(1 \mu \mathrm{M})$ followed by TTX and 4-AP $(100 \mu \mathrm{M})$ to determine monosynaptic connection between the LH GABA neurons and the tVTA/RMTg GABA neurons. 
Miniature inhibitory (mIPSC) postsynaptic currents. Recording electrodes (2-5 M $\Omega$ ) were filled with a $\mathrm{CsCl}$ internal solution. Neurons were voltage clamped at $-70 \mathrm{mV}$ and following the application of tetrodotoxin (TTX; $1 \mu \mathrm{M})$, the current was recorded for 5 min. mIPSCs were identified and measured using Mini Analysis 60 (Synaptosoft, Decateur, GA, USA) and the following parameters: amplitude $>10 \mathrm{pA}$, decay time $<10 \mathrm{~ms}$, and rise time $<4 \mathrm{~ms}$.

Strontium experiments: Recording electrodes were filled with $\mathrm{CsCl}$ internal solution. The 2.4 $\mathrm{mM} \mathrm{CaCl}_{2}$ in the aCSF was replaced with $2.4 \mathrm{mM} \mathrm{SrCl}_{2}$ when slices were transferred from the NMDG solution. Neurons were voltage clamped at $-70 \mathrm{mV}$ and currents were evoked with a single optical stimulation at $0.03 \mathrm{~Hz}(3.5 \mathrm{~mW})$ in the presence of $T \mathrm{TX}$ and 4-AP. To ensure that neurons analyzed received inputs with adequate ChR2 expression, neurons with a current amplitude less than 30 pA immediately following optical stimulation were excluded. Using Mini Analysis 60 and the following parameters: amplitude $>10 \mathrm{pA}$, decay time $<10 \mathrm{~ms}$, and rise time $<4 \mathrm{~ms}$, quantal currents were identified for the first $1000 \mathrm{~ms}$ following the optical stimulation. The amplitude and frequency of $\mathrm{Sr}^{2+}$ events were compared between early (50 to $350 \mathrm{~ms}$ ) and late (700 to $1000 \mathrm{~ms}$ ) periods of asynchronous activity following optical stimulation.

Optical stimulation trains: To evoke olPSCs, recording electrodes (2-5 M 2 ) were filled with a $\mathrm{CsCl}$ internal solution. Neurons were voltage clamped at $-70 \mathrm{mV}$ and either a $20 \mathrm{~Hz}$ over $1 \mathrm{~s}$ (3.5 $\mathrm{mW}$ ) or $0.1 \mathrm{~Hz}$ optical stimulation was used to evoke IPSC trains. A 1 minute, 6 sweep, period was used to create average traces with MatLab (https://github.com/borglandlab/Godfrey-Qiao- 
and-Borgland-2021.git) and current peaks were identified. To ensure that neurons analyzed received inputs with adequate ChR2 expression, neurons with an average first peak amplitude less than 50 pA were excluded. Peak amplitude of the first oIPSC, the readily releasable pool (RRP) and the steady-state oIPSC amplitude were calculated using methods established previously (Thanawala \& Regehr, 2013, 2016). For the $\mathrm{RRP}_{\text {train, }}$ averaged oIPSC amplitudes were summed throughout the train stimulus to give a cumulative oIPSC curve. A line was fit to the final 6 points of the cumulative oIPSC and back extrapolated to the $y$-axis. The slope of this line is the steady-state oIPSC amplitude, reflecting the rate of vesicle recycling after the RRP has been depleted, and the $\mathrm{y}$-intercept is the $\mathrm{RRP}_{\text {train }}$ amplitude. Two neurons were excluded from the RRP analysis as the calculation of the y-intercept produced a negative $y$-intercept, which is physiologically not possible. The paired pulse ratio for the first 5 optical stimulations of the train was calculated as pulse (P) n / pulse (P) 1 (McGarry \& Carter, 2016, 2017).

Current Clamp Recordings: For all current clamp recordings, recording electrodes (2-5 M 2 ) were filled with Potassium-gluconate internal solution containing (in mM): 130 K-gluconate, 10 $\mathrm{KCl}$, 10 HEPES, 0.5 EGTA, $10 \mathrm{Na}_{2}$-Phosphocreatine, 4 MgATP, $0.3 \mathrm{NaGTP}$, and 0.2\% Biocytin. The liquid junction potential was $15.9 \mathrm{mV}$. Recording electrodes (2-5 $\mathrm{M} \Omega$ ) were filled with a Kgluconate internal solution. Current was injected to hold the cell at -70 mV and $500 \mathrm{~ms}$ current steps, from -75 pA to 200 pA in 25 pA increments at $0.1 \mathrm{~Hz}$, were used to evoke action potentials. For the $-75 \mathrm{mV}$ to $0 \mathrm{mV}$ steps, the membrane potential at the end of each step was plotted against the current step. The input resistance was determined to be the slope of the line through these points. Action potentials were identified using Clampfit. Neurons that failed 
to fire action potentials by a $100 \mathrm{pA}$ current step, or with action potentials less that $60 \mathrm{mV}$ were deemed to be unhealthy and were excluded from analysis. To measure the resting membrane potential, the membrane potential of the cell was averaged over 5 minutes using Clampfit. Neurons with spontaneous action potentials were excluded from this calculation.

For optical stimulation during current clamp recordings, current was injected to hold the cell at $-70 \mathrm{mV}$ and $1 \mathrm{~s}$ current steps to $100 \mathrm{pA}$ at $0.1 \mathrm{~Hz}$ were used to evoke action potentials. After allowing the cell to stabilize, a 1-minute (6 sweeps) baseline was recorded. Following the baseline, a $20 \mathrm{~Hz}$ optogenetic stimulation was synchronized with the onset of the current step and the membrane potential was recorded for 1 minute ( 6 sweeps). Using MatLab, action potentials were identified. The latency to fire (time to first peak), peak amplitude (difference between threshold and peak), and the after-hyperpolarization potential (AHP; difference between the inflection point of the AP on the falling phase and threshold voltage) were also calculated and averaged over 1 s or the first 200 ms with or without optical stimulation. Neurons that did not have more than 1 action potential in the first 200 ms of the current step were excluded from this analysis. Depolarization block occurred in neurons that ceased firing in the final 200 ms of the current step. A two-tailed binomial test, Wilson/Brown, was used to compare between the proportion of depolarization block and no depolarization block with and without optogenetic stimulation.

\section{Immunohistochemistry}


Mice were deeply anesthetized with isoflurane and transcardially perfused with phosphate buffered saline (PBS) and then with 4\% paraformaldehyde (PFA). Brains were dissected and post-fixed in $4 \%$ PFA at $4^{\circ} \mathrm{C}$ overnight, then switched to $30 \%$ sucrose. Frozen sections were cut at $30 \mu \mathrm{m}$ using a cryostat. $10 \%$ goat and donkey serums were applied to block non-specific binding for 1 hour. To examine colocalization of TdTomato in VGAT ${ }^{\text {cre }}$ TdTomato mice with tyrosine hydroxylase (TH), horizontal sections were then incubated with primary antibody mouse anti TH 1:1000 (Sigma, T1299) and rabbit red fluorescent protein (RFP) 1:2000 (Rockland, 600-401-379) to amplify the tdTomato signal in 1\% BSA for 24 hours at room temperature followed by incubation with secondary antibody Alexa Fluor 488 goat anti mouse 1:400, Alexa Fluor 594 donkey anti-rabbit 1:400, and DAPI 1:2000 for 1 hour. To check viral transfection, coronal sections were incubated with primary antibody chicken GFP 1:1000 (AVES Labs, GFP-1020) and rabbit RFP (1:2000) in 1\% BSA for 1 hour at room temperature followed by incubation with secondary antibody Alexa Fluor 488 goat anti chicken 1:400 and Alexa Fluor 594 donkey anti-rabbit 1:400 for 1 hour. Slices were mounted with Fluoshield (Sigma). All images were obtained on an Olympus virtual slide microscopy VS120-L100-W with a 10x objective (Olympus Canada Inc., Ontario, Canada) and a Leica confocal microscopy TCS SP8 with a 25x objective (Leica Microsystems Inc., Ontario, Canada). Images were processed using ImageJ/FIJI. Neurons were then counted using the colocalization object counter ImageJ/FIJI plugin and following protocol established by Lunde and Glover (Lunde \& Glover, 2020). Neurons were identified as VGAT+ by co-expression of $\mathrm{td}$-Tomato and DAPI; TH+ by co-expression of green fluorescent protein and DAPI; and VGAT+ with TH+ by co-expression of td-Tomato, green fluorescent protein, and DAPI. To determine if TdTomato fluorescence and TH-like staining co- 
localized, we averaged cell counts from 63 slices from 6 mice, with an average of 21 neurons expressing td-Tomato and DAPI per slice.

Following the voltage clamp $20 \mathrm{~Hz}$ train stimulation experiments, the slices were immediately placed in $4 \%$ PFA to fix for a minimum of 24 hours at $4^{\circ} \mathrm{C}$. The slices were then transferred to PBS and stored at $4^{\circ} \mathrm{C}$. Prior to imaging, slices were rinsed 3 times in PBS and then mounted with Fluoshield (Sigma). Whole brain images were obtained on an Olympus virtual slide microscopy VS120-L100-W with a 10x objective (Olympus Canada Inc., Ontario, Canada) with identical parameters. Using ImageJ, a region of interest was created by tracing the outside of the slice and the value of the mean fluorescent intensity was obtained as an ImageJ measurement.

\section{Data Analysis}

All values are expressed as means +/- SD and assessed for normality using a Shapiro-Wilk test.

In Fig 1 symbols represent individual mice, while in Figs 2-9 symbols represent individual neurons. Outliers were identified and removed using the Robust regression and Outlier removal (ROUT) $(Q=1 \%)$ of current amplitudes and in the change in latency to fire. Statistical significance was assessed by using two-tailed unpaired Student's t test for two comparisons. A two-way ANOVA followed by Sidak's multiple comparisons was used for multiple group comparisons. For time course experiments, either a repeated measures two-way ANOVA followed by Tukey's multiple comparisons test, a mixed effects analysis followed by Tukey's multiple comparisons test, or a repeated measures one-way ANOVA followed by Dunnett's 
multiple comparisons test were used. For comparing proportions, a two-tailed binomial test was used. In all electrophysiology experiments, sample size is expressed as $\mathrm{N} / \mathrm{n}$, where $\mathrm{N}$ refers to the number of neurons recorded from $\mathrm{n}$ animals. Asterisks were used to express statistical significance in figures: ${ }^{*} \mathrm{P}<0.05,{ }^{* *} \mathrm{P}<0.01, * * * \mathrm{P}<0.001$, and $* * * * P<0.0001$. GraphPad Prism 8.3 (GraphPad Software, Inc., La Jolla, CA, USA) was used to perform statistical analysis. Figures were generated using GraphPad Prism 8.3 and Adobe Illustrator CS4 (Adobe Inc., San Jose, CA, USA) software.

\section{Results}

To identify GABAergic neurons in the tVTA/RMTg for electrophysiological recordings, we crossed VGAT ${ }^{\text {cre }}$ mice with Rosa-td Tomato mice. The VTA contains a heterogenous population of neurons, including neurons that contain and release dopamine, GABA, and glutamate (NairRoberts et al., 2008; Chieng et al., 2011). Caudally to the VTA, the neuronal population is primarily GABAergic (Barrot et al., 2012; Bourdy \& Barrot, 2012). To validate our VGAT ${ }^{\text {cre }}$ TdTomato mouse line, we examined colocalization between TdTomato fluorescence, a marker for GABAergic neurons and tyrosine hydroxylase (TH), a marker for dopamine neurons. Of all neurons expressing Td-Tomato, $2.6 \pm 6.7 \%$ co-express $\mathrm{TH}$, with the remaining $97.3 \pm 6.7 \%$ expressing only Td-Tomato (Figure 2A,B), suggesting that the fluorescent label is expressed in non-dopaminergic neurons primarily medial to the medial lemiscus in the VTA. We focused our recordings to the VGAT ${ }^{\text {cre }}$ TdTomato expressing neurons in caudal VTA slices, consistent with the GABAergic population of the tVTA/RMTg (Barrot et al., 2012). 


\section{Effects of fasting on bodyweight and water consumption.}

To determine the effects of a $16 \mathrm{~h}$ overnight fast on VGAT ${ }^{\text {cre }} \mathrm{Td}$-Tomato mice, we measured changes in body weight as well as food and water consumption (Figure 1C). Baseline body weights measured prior to the $16 \mathrm{~h}$ overnight fast were not different between fasted and control groups (fasting effect: $F(1,75)=0.01, P=0.9$ ), but different between sexes (sex effect: $F(1,75)=117.1, P<0.0001$; male control $(n=18): 31.8 \pm 3.5$ g, male fasted $(n=20): 31.4 \pm 4.1$ g, female control $(n=21): 23.0 \pm 2.5$ g, female fasted $(n=20): 23.2 \pm 3.7$ g; Figure 1D). Similar to a previous study (Godfrey \& Borgland, 2020), female control mice consumed more energy than males during the $16 \mathrm{~h}$ period $\left(\mathrm{t}(37)=5.5, \mathrm{P}<0.0001\right.$; male control $(\mathrm{n}=18): 0.5 \pm 0.09 \mathrm{kcal} \mathrm{g}^{-1}$; female control $(n=21): 0.7 \pm 0.1 \mathrm{kcal} \mathrm{g}^{-1}$; Figure $\left.1 \mathrm{E}\right)$. Fasting induced significant weight loss (fasting effect: $F(1,75)=703.7, P<0.0001$; male control $(n=18): 0.6 \pm 2.03 \%$; female control $(n$ $=21): 2.3 \pm 2.4 \%$; male fasted $(n=20):-10.0 \pm 1.6 \%$; female fasted $(n=20):-11.4 \pm 2.0 \%$;

Figure 1F). Weight loss was also affected by sex ( $\operatorname{sex}$ effect: $F(1,75)=0.1, P=0.7)$ with both male $(P<0.0001)$ and female $(P<0.0001)$ fasted mice losing more weight than their controls, as indicated by Sidak's multiple comparisons test (sex $x$ fasting interaction: $(F(1,75)=11.0$, $P=0.0014)$. There was a main effect of fasting on water consumption, with fasted mice consuming less water than controls (fasting effect: $F(1,75)=408.3, P<0.0001$; male control $(n=$ 18): $3.0 \pm 0.6 \mathrm{~mL}$; female control $(n=21): 3.3 \pm 0.4 \mathrm{~mL}$; male fasted $(n=20): 1.0 \pm 0.4 \mathrm{~mL}$; female fasted $(n=20): 1.1 \pm 0.5 \mathrm{~mL}$; Figure $1 \mathrm{G})$. However, there was no sex difference in water consumption (sex effect: $F(1,75)=2.2, P=0.1$ ). Taken together, a $16 \mathrm{~h}$ fast induces weight loss in male and female mice. 


\section{Effects of fasting on tVTA/RMTg GABA neurons}

To test the effect of fasting on GABAergic synaptic transmission onto tVTA/RMTg GABAergic neurons, we recorded miniature inhibitory postsynaptic currents (mIPSCs). There were no main effects of fasting or sex (fasting effect: $F(1,71)=0.2, P=0.6$; sex effect: $F(1,71)=3.8, P=0.05$ ). However, we observed a significant cross-over interaction between fasting and sex on mIPSC amplitude (sex $x$ fasting interaction: $F(1,71)=5.15, P=0.03$; male control $(N / n=16 / 4): 73.4 \pm$ 30.4 pA, female control $(N / n=20 / 4): 47.9 \pm 23.6 p A ;$ male fasted $(N / n=18 / 5): 56.8 \pm 24.3 p A$; female fasted $(\mathrm{N} / \mathrm{n}=21 / 4): 58.7 \pm 26.0 \mathrm{pA}$; Figure $2 \mathrm{C})$. A Sidak's posthoc test indicates there was a significant difference between male and female control mIPSC amplitude ( $P=0.02)$. To explore the GABAergic release onto tVTA/RMTg GABAergic neurons, we next examined mIPSC frequency. A main effect of sex was observed on mIPSC frequency (sex effect: $F(1,71)=5.3$, $\mathrm{P}=0.02$; male control $(\mathrm{N} / \mathrm{n}=16 / 4): 5.4 \pm 4.3 \mathrm{~Hz}$; female control $(\mathrm{N} / \mathrm{n}=20 / 4): 3.8 \pm 3.7 \mathrm{~Hz}$; male fasted $(N / n=18 / 5): 6.2 \pm 5.9 \mathrm{~Hz}$; female fasted $(\mathrm{N} / \mathrm{n}=21 / 4): 3.3 \pm 2.7 \mathrm{~Hz}$; Figure 2D). However, there was no effect of fasting (fasting effect: $F(1,71)=0.009, P=0.9$ ) or sex $x$ fasting interaction (Interaction: $\mathrm{F}(1,71)=0.5, \mathrm{P}=0.5)$. Taken together, these results suggest that there were sex differences in GABAergic synaptic transmission onto tVTA/RMTg GABAergic neurons, but this is not strongly affected by fasting.

We next examined how fasting alters the excitability of tVTA/RMTg GABAergic neurons using current steps from -75 pA to 200 pA in 25 pA increments at $0.1 \mathrm{~Hz}$ while neurons were initially held at -70 mV. Analysis of the frequency-current (F-I) plot revealed a main effect of current steps (current effect: $F(7.0,378.0)=21.3 ; P<0.0001$ ) and a main effect of group (group effect: $F$ 
$(3.0,378.0)=5.6, P=0.0009$, Figure $3 C)$ with both male $(P=0.02)$ and female $(P=0.03)$ fasted

being less excitable than male and female controls, as indicated by a Tukey's multiple comparisons test. There was no effect of fasting or sex on the input resistance (fasting effect: $F$ $(1,35)=0.1, P=0.7 ;$ sex effect: $F(1,35)=0.7, P=0.4 ;$ male control $(N / n=10 / 4): 0.6 \pm 0.3$ mOhms; female control $(N / n=9 / 3): 0.6 \pm 0.3 \mathrm{mOhms}$, male fasted $(\mathrm{N} / \mathrm{n}=12 / 5): 0.7 \pm 0.2$ mOhms; female fasted $(\mathrm{N} / \mathrm{n}=8 / 4): 0.6 \pm 0.4 \mathrm{mOhms;}$ Figure 3D). However, a main effect of fasting was observed in the resting membrane potential (fasting effect: $F(1,42)=4.8, P=0.03$; male control $(\mathrm{N} / \mathrm{n}=9 / 6):-69.7 \pm 3.4 \mathrm{mV}$; female control $(\mathrm{N} / \mathrm{n}=12 / 5):-69.7 \pm 7.3 \mathrm{mV}$; male fasted $(N / n=11 / 6):-66.3 \pm 7.6 \mathrm{mV}$; female fasted $(N / n=14 / 8):-64.9 \pm 5.8 \mathrm{mV}$; Figure 3E) with fasted mice having a more depolarized resting membrane potential than controls. Taken together, these data suggest that fasting induces changes in excitability as well as resting membrane potential in both sexes.

\section{Effect of fasting on inhibitory synaptic transmission at LH GABA to tVTA/RMTg GABA}

\section{synapses.}

We noted a significant amount of variability in the mIPSC recordings from control and fasted mice and therefore questioned if possible fasting-induced changes in GABAergic synaptic transmission may be driven by a specific input. LH GABAergic neurons become active during appetitive and consummatory behaviours (Jennings et al., 2015) and activation of LH GABA inputs to caudomedial VTA GABAergic neurons increases motivated behaviour for food or social reward (Nieh et al. 2015, 2016). Therefore, we predicted LH GABAergic inputs to the tVTA/RMTg may be susceptible to changes induced by fasting. We first verified the expression 
of ChR2 in LH GABAergic neurons (Figure 4A). We then recorded action potentials of LH GABAergic neurons evoked by optical stimulation $(470 \mathrm{~nm})$. In current clamp, action potentials were reliably evoked in LH GABAergic neurons with $5 \mathrm{~Hz}, 10 \mathrm{~Hz}$, and $20 \mathrm{~Hz}$ optical stimulation (Figure 4B). Similarly, in voltage clamp of tVTA/RMTg GABAergic neurons, IPSCs were reliably evoked by $5 \mathrm{~Hz}, 10 \mathrm{~Hz}$, and $20 \mathrm{~Hz}$ optical stimulation (Figure 4C). A 1 second stimulation produced a sustained depolarization in current clamp of LH GABAergic neurons, whereas this optical stimulation produced a single IPSC at tVTA/RMTg GABAergic neurons (Figure 4D, E). We next tested the nature of the LH GABA to tVTA/RMTg GABA input (Figure 4F). Optical stimulation of LH inputs evoked IPSCS onto tVTA/RMTg GABAergic neurons and this effect was blocked by picrotoxin $(t(4)=4.4$; baseline: $-376.7 \pm 191.1 \mathrm{pA}$, picrotoxin: $-8.1 \pm 5.3 \mathrm{pA}, \mathrm{N} / \mathrm{n}=5 / 2$; Figure 4G). To test if this projection was monosynaptic, we applied the sodium channel blocker, TTX and then assessed if the current returned in the presence of 4-AP. TTX blocked optically evoked IPSCs (oIPSCs; baseline: $-781.1 \pm 317.9$ pA, TTX: $-39.6 \pm 48.2 \mathrm{pA}, \mathrm{N} / \mathrm{n}=5 / 3$ ), and this was restored by 4-AP $(-741.4 \pm 493.2 \mathrm{pA}, \mathrm{N} / \mathrm{n}=5 / 3$; one-way ANOVA: $(\mathrm{F}(1.7,6.7)=11.3, \mathrm{P}=0.008$; Dunnett's multiple comparisons tests baseline vs. TTX: $P=0.009$; baseline vs. $T \mathrm{X}+4-\mathrm{AP}: \mathrm{P}=0.9$; Figure 4H). Taken together, these data suggest that LH GABAergic neurons functionally synapse onto tVTA/RMTg GABAergic neurons though a monosynaptic projection.

To probe the quantal properties of LH GABAergic input to tVTA/RMTg GABA neurons, extracellular $\mathrm{Ca}^{2+}$ was replaced by $\mathrm{Sr}^{2+}$ leading to asynchronous exocytosis of vesicles allowing the resolution of quantal synaptic events. Under these experimental conditions, optogenetic stimulation of LH GABAergic inputs to tVTA/RMTg GABA neurons evoked asynchronous release 
identified by quantal-like events (qIPSCs). In the first $50 \mathrm{~ms}$, the optical stimulation was

frequently followed by a summation of synchronized events that interfere with identification of asynchronous qIPSCs. Hence, we followed previously established protocols and analyzed 50 to 350 ms following the optical stimulation for asynchronous qIPSCs (MacAskill et al., 2014;

Geddes et al., 2016). We also identified qIPSCs between 700 to 1000 ms following the optical stimulation, a period where the effects of the optical stimulation have mostly ended. This gave us time periods to examine both the LH GABA input (50 to $350 \mathrm{~ms}$ ) and all inputs (700 to 1000 ms). Between 50 to 350 ms following the optical stimulation, there was a main effect of fasting (fasting effect: $F(1,47)=7.1, P=0.01)$ and a main effect of sex (sex effect: $F(1,47)=5.5$, $\mathrm{P}=0.02)$ on qIPSC amplitudes (male control $(\mathrm{N} / \mathrm{n}=14 / 4): 76.4 \pm 34.2 \mathrm{pA}$; female control $(\mathrm{N} / \mathrm{n}=$ 13/5): $57.4 \pm 22.9 \mathrm{pA}$, male fasted $(\mathrm{N} / \mathrm{n}=13 / 4): 55.3 \pm 13.5 \mathrm{pA}$; female fasted $(\mathrm{N} / \mathrm{n}=11 / 5)$ :

43.2 $\pm 16.1 \mathrm{pA}$; Figure 5B). However, between 700 to $1000 \mathrm{~ms}$ following the optical stimulation there was neither a main effect of fasting (fasting effect: $F(1,47)=0.02, P=0.9$ ) or sex ( $\operatorname{sex}$ effect: $F(1,47)=0.001, P=1.0)$ on qIPSC amplitude (male control $(N / n=14 / 4): 48.2 \pm 18.3 p A$; female control $(N / n=13.5): 49.4 \pm 17.3 p A$, male fasted $(N / n=13 / 4): 50.4 \pm 26.7 p A ;$ female fasted $(\mathrm{N} / \mathrm{n}=11 / 5): 48.9 \pm 11.6 \mathrm{pA}$; Figure $5 \mathrm{C})$.

Frequency of qIPSCs between 50 to 350 ms following the optical stimulation was significantly different in fasted mice (Fasting effect: $F(1,47)=6.4, P=0.02$ ) but no effect of sex (sex effect: $F$ $(1,47)=0.2, P=0.6)$, or a sex $x$ fasting interaction $(F(1,47)=1.6, P=0.2)$. A Sidak's multiple comparisons test on the effect of fasting revealed no difference in frequency between male control $(14.4 \pm 11.7 \mathrm{~Hz}, \mathrm{~N} / \mathrm{n}=14 / 4)$ and fasted mice $(11.2 \pm 6.9 \mathrm{~Hz}, \mathrm{~N} / \mathrm{n}=13 / 4 ; \mathrm{P}=0.6)$, but a 
significant difference in female control $(18.9 \pm 9.6 \mathrm{~Hz}, \mathrm{~N} / \mathrm{n}=13 / 5)$ and fasted mice $(9.2 \pm 6.4 \mathrm{~Hz}$, $N / n=11 / 5 ; P=0.02 ;$ Figure 5D). Between 700 to 1000 ms following optical stimulation, there was no main effect of fasting $(F(1,47)=0.8, P=0.4)$, sex effect $(F(1,47)=1.3, P=0.3)$, or sex $x$ fasting interaction on qIPSC frequency (interaction: $F(1,47)=0.00003, P=1.0$; male control $(N / n=14 / 4): 5.4 \pm 3.1 \mathrm{~Hz}$; female control $(N / n=13 / 5): 6.5 \pm 3.1 \mathrm{~Hz}$; male fasted $(N / n=13 / 4)$ : $4.5 \pm 3.8 \mathrm{~Hz}$; female fasted $(\mathrm{N} / \mathrm{n}=11 / 5): 5.6 \pm 4.1 \mathrm{~Hz}$; Figure 5E). Taken together, these data indicate that fasting decreases amplitude of qIPSCs induced by optical stimulation of LH GABA inputs to tVTA/RMTg GABA in all mice, but only decreases qIPSC frequency in female mice.

To characterize properties of short-term synaptic plasticity at these synapses, we next examined if olPSCs from LH GABAergic inputs to tVTA/RMTg GABAergic neurons were altered by fasting using a $20 \mathrm{~Hz}$ optical train stimulation (Figure $6 \mathrm{~A}-\mathrm{C}$ ). We first assessed for changes in vesicle release kinetics determined by the readily releasable pool size $\left(R_{R P}\right.$ train $)$ and steady-state oIPSC amplitude, reflecting the replenishment of the RRP after it has been depleted (Thanawala \& Regehr, 2013, 2016). Analysis of the first oIPSC revealed an effect of fasting on the amplitude of olPSCs onto tVTA/RMTg GABAergic neurons (fasting effect: $F(1,79)=13.1, P=0.0005$; male control $(N / n=21 / 5):-553.2 \pm 462.0 p A ;$ male fasted $(N / n=23 / 5):-281.4 \pm 230.0 p A ;$ female control $(N / n=19 / 5):-640.3 \pm 461.7 p A ;$ female fasted $(N / n=20 / 5):-331.1 \pm 251.3 p A ;$ Figure $6 D)$, but no effect of sex (sex effect: $F(1,79)=0.7, P=0.4)$. There was no significant interaction of sex $x$ fasting $(F(1,79)=0.05, P=0.8)$. In addition, there was a fasting effect on the $R R P_{\text {train }}$ (fasting effect: $F(1,75)=10.3, P=0.002 ;$ male control $(N / n=20 / 5): 1186 \pm 1121 p A$, male fasted $(N / n=23 / 5): 685.8 \pm 615.5 p A$, female control $(N / n=18 / 5): 1692 \pm 1356 p A$, female fasted 
$(N / n=18 / 5): 783.1 \pm 656.6 p A ;$ Figure $6 E, F)$ and the steady-state olPSC amplitude (fasting effect: $F(1,79)=7.3, P=0.009$ pA; male control $(N / n=21 / 5): 241.4 \pm 207.2 p A$, male fasted $(N / n=23 / 5): 99.2 \pm 82.0 p A$, female control $(N / n=19 / 5): 205.8 \pm 193.9 p A$, female fasted $(N / n$ $=20 / 5$ ): $157.6 \pm 136.0$ pA; Figure $6 G$ ). There was no effect of sex or sex $x$ fasting interaction in either the $R R P_{\text {train }}($ sex effect: $F(1,75)=1.9, P=0.2$; fasting $x$ sex: $F(1,75)=0.9, P=0.4$ ) or the steady-state oIPSC amplitude (sex effect: $F(1,79)=0.1, P=0.7$; fasting $x \operatorname{sex}: F(1,79)=1.8$, $P=0.2$ ). Thus, fasting decreased the amplitude of the first pulse, the effective RRP size, and the steady-state oIPSC amplitude of the LH GABAergic input to tVTA/RMTg GABAergic neurons in male and female mice.

To determine if there are fasting-induced differences in short-term plasticity, we measured the ratio of the amplitude of oIPSCS (Pn) over the first oIPSC (P1) for each of the first 5 pulses. While male mice exhibited a paired-pulse depression (pulse effect: $F(2.3,94.9)=23.4, P<0.0001$ ), there was no effect of fasting (fasting effect: $F(1,42)=0.03, P=0.9$; Figure 6I). However, there was a significant paired pulse depression in female mice (pulse effect: $F(2.8,104.9)=18.6, P<0.0001$ ), and an effect of fasting (fasting effect: $F(1,37)=5.09, P=0.03$; Figure $6 \mathrm{~L}$ ). Taken together, fasting alters the release probability of LH GABA inputs of female mice.

To address the potential of differences in ChR2 expression between groups, we quantified eYFP fluorescence intensity from slices used for the $20 \mathrm{~Hz}$ train optical stimulation recordings. There were no fasting effects (fasting effect: $F(1,51)=0.04, P=0.8$ ) or sex effects on eYFP fluorescence intensity (sex effect: $F(1,51)=3.07, P=0.08$; fasting $x$ sex: $F(1,51)=0.6, P=0.4)$; 
male control $(\mathrm{N} / \mathrm{n}=13 / 5): 4943 \pm 1197$, male fasted $(\mathrm{N} / \mathrm{n}=13 / 5): 5314 \pm 1174$, female control $(N / n=14 / 5): 4583 \pm 1839$, female fasted $(N / n=15 / 5): 4365 \pm 1190 ;$ Figure $6 H)$. Thus,

presumably ChR2 expression at LH GABA inputs to the VTA is similar between groups and not driving electrophysiological differences.

\section{Effect of fasting on firing rate of tVTA/RMTg GABA neurons during optical stimulation of LH}

\section{GABA inputs.}

We next tested whether the effects of a $20 \mathrm{~Hz}$ optical stimulation on synaptic transmission of LH GABAergic terminals influenced evoked firing of tVTA/RMTg GABAergic neurons. We analyzed firing properties of tVTA/RMTg GABAergic neurons over the entire $1 \mathrm{~s}$ step, as well as just the first $200 \mathrm{~ms}$, which is the duration of 5 pulses at $20 \mathrm{~Hz}$. In males, over the 1s step, there was no effect of optical stimulation or fasting on number of action potentials (stimulation effect: $F(1,20)=0.02, P=0.9$; fasting (fasting effect: $F(1,20)=0.03, P=0.9$; fasting $\times$ stimulation: $F(1,20)=0.4, P=0.5 ;$ male control pre-OPTO $(N / n=12 / 6): 14.0 \pm 16.1$, male fasted pre-OPTO $(\mathrm{N} / \mathrm{n}=10.6): 13.5 \pm 10.2$, male control OPTO $(\mathrm{N} / \mathrm{n}=12 / 6): 12.31 \pm 13.16$, male fasted OPTO $(N / n=10.6): 14.5 \pm 13.5$; Figure 7A-D). In female mice, over the $1 s$ step there was an effect of optical stimulation on action potentials (stimulation effect: $F(1,18)=5.8, P=0.03$; female control pre-OPTO $(\mathrm{N} / \mathrm{n}=10 / 5): 14.3 \pm 12.5$, female fasted pre-OPTO $(\mathrm{N} / \mathrm{n}=10 / 7): 21.6 \pm 22.8$, female control with OPTO $(\mathrm{N} / \mathrm{n}=10 / 5): 8.6 \pm 11.5$, female fasted with OPTO $(\mathrm{N} / \mathrm{n}=10 / 7): 21.0$ \pm 22.3 ; Figure 7G-I). However, like male mice, there was no effect of fasting on action potentials in female mice (fasting effect: $F(1,18)=1.5, P=0.2$; fasting $x$ stimulation: $F(1,18)=2.6, P=0.1$; Figure 7F-H). Within the first 200 ms of the current step, there was an effect of optical 
stimulation on action potentials for both males (stimulation effect: $F(1,20)=15.6, P=0.0008$;

male control pre-OPTO $(\mathrm{N} / \mathrm{n}=12 / 6): 6.7 \pm 4.7$, male fasted pre-OPTO $(\mathrm{N} / \mathrm{n}=10 / 6): 6.3 \pm 4.6$, male control with OPTO $(\mathrm{N} / \mathrm{n}=12 / 6): 4.8 \pm 4.3$, male fasted with OPTO $(\mathrm{N} / \mathrm{n}=10 / 6): 5.1 \pm 4.3$; Figure $7 E$ ) and females (stimulation effect: $F(1,18)=8.7, P=0.009$; female control pre-OPTO $(\mathrm{N} / \mathrm{n}=10 / 5): 6.1 \pm 4.7$, female fasted pre-OPTO $(\mathrm{N} / \mathrm{n}=10 / 7): 7.4 \pm 4.7$, female control OPTO $(N / n=10 / 5): 3.6 \pm 2.9$, female fasted OPTO $(N / n=10 / 7): 6.7 \pm 4.9$; Figure $7 \mathrm{I})$. However, there was no effect of fasting in either males (fasting effect: $F(1,20)=0.03, P=0.9$; fasting $x$ stimulation: $F(1,20)=0.4, P=0.4)$ or females $(F(1,18)=1.4, P=0.2$; fasting $\times$ stimulation: $F(1$, $18)=1.5, P=0.2$ ). To further examine if fasting influenced the change in action potential number during the first 5 pulses (200 ms), we examined the change in firing induced by optogenetic stimulation. There was a significant effect of fasting on the percent change in firing (fasting effect: $F(1,38)=5.1, P=0.03$ ), but no effect of sex or interaction (sex effect: $F(1,38)=0.01$, $P=0.9$, sex $x$ fasting interaction: $F(1,38)=0.03, P=0.8$; Figure $7 \mathrm{~J})$. Taken together, optical stimulation LH GABAergic input decreases firing of tVTA/RMTg GABAergic neurons and fasting alters the change in firing rate induced by optogenetic stimulation.

We noticed that depolarization block occurred in a significant proportion of neurons with a $1 \mathrm{~s}$ current step, defined as no action potentials in the final $200 \mathrm{~ms}$ of the current step. Prior to optical stimulation, we found no difference in the occurrence of depolarization block between male and female control mice (male control $(\mathrm{N} / \mathrm{n}=12 / 6): 50 \%$, female control (female control $(N / n=13 / 5): 53.8 \% ; P>0.9999)$. Therefore, we grouped male and female mice together to examine the effects of fasting on optogenetic stimulation on the proportion of neurons with 
depolarization block. In control mice, there was no difference between pre stimulation (control pre-OPTO (N/n = 25/11): 52\%) and optical stimulation (control OPTO (N/n=25/11): 40\%; $\mathrm{P}=$ 0.2380; figure $5 \mathrm{~N}$ ). However, in fasted mice, optical stimulation significantly decreased the proportion of neurons with depolarization block (fasted pre-OPTO (N/n = 26/13): $38.5 \%$, fasted OPTO (N/n = 26/13 ): 19.2\%; P = 0.04; Figure 7K). These data suggest that during fasting, the function of the LH GABA to tVTA/RMTg GABA synapse is preserved under depolarized conditions.

We next examined how optical stimulation influenced action potential characteristics. Consistent with a reduction in action potential number, optical stimulation of LH GABA inputs increased the latency to fire in both males (stimulation effect: $F(1,21)=34.3, P<0.0001$; male control pre-OPTO ( $\mathrm{N} / \mathrm{n}=11 / 6): 19.3 \pm 25.1 \mathrm{~ms}$, male fasted pre-OPTO $(\mathrm{N} / \mathrm{n}=12 / 6): 50.3 \pm 62.4$ ms, male control OPTO (N/n = 11/6): $35.9 \pm 35.6 \mathrm{~ms}$, male fasted OPTO $(\mathrm{N} / \mathrm{n}=12 / 6): 71.7 \pm$ $76.6 \mathrm{~ms}$; Figure $8 \mathrm{~A}$ ) and females (stimulation effect: $\mathrm{F}(1,23)=5.4, \mathrm{P}=0.03$; female control preOPTO $(\mathrm{N} / \mathrm{n}=12 / 5): 56.3 \pm 76.2 \mathrm{~ms}$, female fasted pre-OPTO $(\mathrm{N} / \mathrm{n}=13 / 7): 52.9 \pm 80.2 \mathrm{~ms}$, female control OPTO $(\mathrm{N} / \mathrm{n}=12 / 5): 119.5 \pm 151.2 \mathrm{~ms}$, female fasted OPTO $(\mathrm{N} / \mathrm{n}=13 / 7): 73.9 \pm$ 107.0 ms; Figure 8B). No effect of fasting was observed in either males (fasting effect: $F(1,21)=$ 2.1, $P=0.1$, fasting $\times$ stimulation: $F(1,21)=0.5, P=0.5)$ or females (fasting effect: $F(1,23)=0.4$, $P=0.5$; fasting $x$ stimulation: $F(1,23)=1.3, P=0.3)$. However, there was a significant effect of fasting on the percent change in latency to fire (fasting effect: $F(1,42)=4.97, P=0.03$ ), but no effect of sex or interaction (sex effect: $F(1,42)=3.39, P=0.07$, sex $x$ fasting interaction: $F(1,42$ ) $=0.035, \mathrm{P}=0.85$; Figure $8 \mathrm{C}$ ). There was no change in AHP due to $20 \mathrm{~Hz}$ optical stimulation 
analyzed over the $1 \mathrm{~s}$ current step in either males (stimulation effect: $F(1,19)=2.1, P=0.2$;

male control pre-OPTO $(\mathrm{N} / \mathrm{n}=11 / 6): 15.2 \pm 3.7 \mathrm{mV}$, male control OPTO $(\mathrm{N} / \mathrm{n}=11 / 6): 15.2 \pm 4.4$ $\mathrm{mV}$, male fasted pre-OPTO $(\mathrm{N} / \mathrm{n}=10 / 6): 16.4 \pm 5.9 \mathrm{mV}$, male fasted OPTO $(\mathrm{N} / \mathrm{n}=10 / 6): 18.1 \pm$ $6.3 \mathrm{mV}$; Figure $8 \mathrm{C}, \mathrm{E}$ ) or females (stimulation effect: $\mathrm{F}(1,18)=0.5, \mathrm{P}=0.5$; female control preOPTO $(\mathrm{N} / \mathrm{n}=10 / 5): 16.7 \pm 4.1 \mathrm{mV}$, female control OPTO $(\mathrm{N} / \mathrm{n}=10 / 5): 17.6 \pm 5.1 \mathrm{mV}$, female fasted pre-OPTO $(\mathrm{N} / \mathrm{n}=10 / 7): 15.3 \pm 5.0 \mathrm{mV}$, female fasted OPTO (N/n = 10/7): $15.1 \pm 4.9 \mathrm{mV}$; Figure $8 \mathrm{H}, \mathrm{J})$. There was no effect of fasting in the $1 \mathrm{~s}$ current step observed in either males (fasting effect: $F(1,19)=0.9, P=0.3$; fasting $\times$ stimulation: $F(1,19)=2.040, P=0.1694 ;$ Figure $8 E$ ) or females (fasting effect: $F(1,18)=0.9, P=0.4$; fasting $x$ stimulation: $F(1,18)=0.9, P=0.3$; Figure 8I). When analyzing the first $200 \mathrm{~ms}$ of the current step, optical stimulation had no effect on the AHP in either males (stimulation effect: $F(1,19)=2.9, P=0.1$; male control pre-OPTO $(\mathrm{N} / \mathrm{n}=11 / 6): 14.6 \pm 4.4 \mathrm{mV}$, male control OPTO $(\mathrm{N} / \mathrm{n}=11 / 6): 15.2 \pm 4.4 \mathrm{mV}$, male fasted preOPTO $(\mathrm{N} / \mathrm{n}=10 / 6): 16.7 \pm 6.2 \mathrm{mV}$, male fasted OPTO $(\mathrm{N} / \mathrm{n}=10 / 6): 18.0 \pm 6.5 \mathrm{mV}$; Figure $8 \mathrm{G})$ or females (stimulation effect: $F(1,18)=0.8, P=0.4$; female control pre-OPTO $(N / n=10 / 5): 16.9 \pm$ $4.3 \mathrm{mV}$, female control OPTO $(\mathrm{N} / \mathrm{n}=10 / 5): 17.6 \pm 5.2 \mathrm{mV}$, female fasted pre-OPTO $(\mathrm{N} / \mathrm{n}=10 / 7)$ : 15.7 $\pm 5.4 \mathrm{mV}$, female fasted OPTO $(\mathrm{N} / \mathrm{n}=10 / 7): 15.7 \pm 5.4 \mathrm{mV}$; Figure $8 \mathrm{~K})$. Furthermore, there was no effect of fasting on AHP observed in either males (fasting effect: $F(1,19)=1.2, P=0.3$; fasting $x$ stimulation: $F(1,19)=0.5, P=0.5$ ) or females (fasting effect: $F(1,18)=0.5, P=0.5$; fasting $x$ stimulation: $F(1,18)=0.7, P=0.4)$. Taken together, these data suggest that the decrease in firing of tVTA/RMTg GABA neurons due to optogenetic stimulation of LH GABA inputs is mediated by an increased latency to fire rather than a change in AHP. 
We next examined the effect of optical stimulation of LH GABAergic inputs and fasting on action potential height. There was no effect of optical stimulation over the 1 s current step on action potential height in males (stimulation effect: $F(1,19)=1.7, P=0.2$; male control preOPTO (N/n = 11/6): $83.3 \pm 9.5 \mathrm{mV}$, male control OPTO $(\mathrm{N} / \mathrm{n}=11 / 6): 84.1 \pm 11.6 \mathrm{mV}$, male fasted pre-OPTO (N/n = 10/6): $82.5 \pm 8.6 \mathrm{mV}$, male fasted OPTO $(\mathrm{N} / \mathrm{n}=10 / 6): 84.1 \pm 8.3 \mathrm{mV}$; Figure $9 \mathrm{~A}-\mathrm{C}$ ) or females (stimulation effect: $\mathrm{F}(1,18)=2.4, \mathrm{P}=0.1$; female control pre-OPTO $(\mathrm{N} / \mathrm{n}=$ 10/5): $76.7 \pm 7.1 \mathrm{mV}$, female control OPTO (N/n = 10/5): $79.6 \pm 7.1 \mathrm{mV}$, female fasted pre-OPTO $(N / n=10 / 7): 75.0 \pm 6.3 \mathrm{mV}$, female fasted OPTO $(\mathrm{N} / \mathrm{n}=10 / 7): 74.9 \pm 6.6 \mathrm{mV}$; Figure $9 \mathrm{E}-\mathrm{G})$ Fasting did not alter action potential height over the 1s current step in either males (fasting effect: $F(1,19)=0.006, P=0.9$; fasting $x$ stimulation: $F(1,19)=0.2, P=0.7)$ or females (fasting effect: $F(1,18)=1.2, P=0.3$; fasting $\times$ stimulation: $F(1,18)=2.8, P=0.1)$. However, when analyzing the first $200 \mathrm{~ms}$ of the current step, we found that the optical stimulation increased the action potential height in both males (stimulation effect: $F(1,19)=6.6, P=0.02$; male control pre-OPTO $(\mathrm{N} / \mathrm{n}=11 / 6): 83.9 \pm 9.6 \mathrm{mV}$, male control OPTO $(\mathrm{N} / \mathrm{n}=11 / 6): 85.6 \pm 9.8 \mathrm{mV}$, male OPTO $(\mathrm{N} / \mathrm{n}=10 / 6): 84.5 \pm 10.0 \mathrm{mV}$, male fasted OPTO $(\mathrm{N} / \mathrm{n}=10 / 6): 85.5 \pm 9.9 \mathrm{mV}$; Figure 9D) and females (stimulation effect: $F(1,18)=4.9, P=0.04$; female control pre-OPTO $(N / n=$ 10/5): $79.1 \pm 6.9 \mathrm{mV}$, female control OPTO ( $\mathrm{N} / \mathrm{n}=10 / 5): 80.7 \pm 7.2 \mathrm{mV}$, female fasted pre-OPTO $(\mathrm{N} / \mathrm{n}=10 / 7): 77.5 \pm 7.1 \mathrm{mV}$, female fasted OPTO $(\mathrm{N} / \mathrm{n}=10 / 7): 77.8 \pm 7.1 \mathrm{mV} ;$ Figure $9 H)$. There was no effect of fasting on action potential height in the first $200 \mathrm{~ms}$ of the current step in either males (fasting effect: $F(1,19)=0.003, P=0.9$; fasting $\times$ stimulation: $F(1,19)=0.5, P=0.5$ ) or females (fasting effect: $F(1,18)=0.5, P=0.5$; fasting $x$ stimulation: $F(1,18)=2.0, P=0.2$ ). 
Thus, optical stimulation of LH GABAergic inputs only alters the action potential height within the first 200 ms of the current step in male or female mice.

\section{Discussion}

Here, we found that fasting decreased the excitability of tVTA/RMTg GABAergic neurons, suggesting that this population of neurons is also sensitive to motivational state. LH GABAergic neurons make a monosynaptic projection to tVTA/RMTg GABAergic neurons. Inhibitory synaptic transmission of this input onto tVTA/RMTg GABAergic neurons was sensitive to fasting, such that fasting decreased the release probability at LH GABAergic terminals of female mice and decreased the amplitude of optically induced currents, RRP size, and RRP replenishment in both male and female mice. Finally, activation of the LH GABAergic input to the VTA decreased firing of tVTA/RMTg GABAergic neurons, and this effect was minimized in fasted mice. These results suggest that fasting can reduce the excitability of tVTA/RMTg GABAergic neurons and the inhibitory effect of the LH GABAergic input to these neurons, leading to preservation of the function of this synapse. Because LH GABAergic neurons can disinhibit VTA dopamine neurons leading to increased NAC dopamine and behavioural activation (Nieh et al., 2016), activation of weaker LH GABA inputs, following fasting, may still be sufficient to induce suppression of tVTA/RMTg GABAergic neuronal activity to allow for foraging.

Consistent with our previous work (Godfrey \& Borgland, 2020), a 16h overnight fast reduced body weight in male and female mice. Previous studies have reported that this reduction in weight is due to both a reduction in body weight (60\%) and gastric emptying (40\%) (Prior et al., 
2012). Although we did not see sex differences in weight change, our previous findings demonstrated that, as a physiological stressor, fasting affects males and females differently, with females observed to have a higher concentration of corticosterone (Godfrey \& Borgland, 2020). Furthermore, given that this duration of fast can increase food approach behaviours and food intake after fasting (Godfrey and Borgland, 2020), this likely represents a motivational state of hunger.

Sex differences of GABAergic synaptic transmission onto tVTA/RMTg neurons

In unfasted control mice, we observed sex differences in inhibitory synaptic transmission onto tVTA/RMTg GABAergic neurons. Female mice had decreased amplitude and frequency of mIPSCs or qIPSCs onto tVTA/RMTg GABAergic neurons compared to male mice. This could be due to a decrease in postsynaptic $\mathrm{GABA}_{\mathrm{A}}$ receptor current size leading to a decrease in mIPSC event detection or due to a decrease in number of inhibitory synapses onto tVTA/RMTg neurons in female mice. Notably, the delta-containing subunit of $\mathrm{GABA}_{\mathrm{A}}$ receptors are regulated by ovarian hormones (Stell et al., 2003; Maguire et al., 2005). Associated Gabrd transcript levels in the VTA are altered across the estrous cycle (Melón et al., 2017) and $\delta$-GABAA receptors can influence lateral diffusion of the receptor across the membrane and its ability to cluster at the synapse, ultimately influencing tonic inhibition (Jacob et al., 2008). Thus, it is possible this difference in inhibitory synaptic transmission could be due to either differential expression of $\delta$ $\mathrm{GABA}_{\mathrm{A}}$ receptors or their regulation by neurosteroids. Alternatively, sex differences in GABAergic synaptic transmission could be due to differences in endocannabinoid modulation of inhibitory inputs to tVTA/RMTg neurons. Notably, tonic endocannabinoid signaling at inhibitory 
inputs to dopamine neurons was greater in female compared to male rats (Melis et al., 2013) suggesting potential sex differences in endocannabinoid signaling within the VTA.

Although fasting did not alter inhibitory synaptic transmission of tVTA/RMTg neurons, fasting suppressed the excitability of tVTA/RMTg neurons of both male and female mice. These changes in excitability were not paralleled with changes in input resistance, suggesting that this change may be driven by changes in the strength of synaptic input rather than intrinsic conductances. It is possible that enhanced tonic GABA release may underlie the fasting-induced decrease in excitability of tVTA/RMTg neurons of male and female mice, as tonic inhibition strongly influences neuronal excitability (Farrant \& Nusser, 2005). These effects were surprising as, unlike fasting, other aversive states such as pain, predator odours, opioid withdrawal and noxious stimuli increase activity of tVTA/RMTg neurons (Sánchez-Catalán et al., 2017; Li et al., 2019a). Notably, the resting membrane potential was more depolarized following fasting; a change that should theoretically produce an increase in excitability. It is possible that there are independent changes in excitatory input that could influence the resting membrane potential. For example, the lateral habenula (LHb) sends excitatory projections to tVTA/RMTg neurons leading to their activation during aversive stimuli (Brown et al. , 2017; Li et al., 2019b).

\section{Sex differences of LH GABA inputs to $\mathrm{tVTA} / \mathrm{RMTg}$ GABA synapses}

LH GABAergic neurons functionally synapse onto tVTA/RMTg GABAergic neurons though a monosynaptic projection. This effect is consistent with previous studies that have recorded from VTA GABAergic interneurons that disinhibit dopamine neurons (Nieh et al., 2015). 
tVTA/RMTg GABA neurons send dense projections to dopamine neurons in the VTA and substantia nigra (SN) (Barrot et al., 2012; Bourdy \& Barrot, 2012). Due to their dense projections and proximal dendritic connections, they have a strong influence over the activity of dopamine neurons and are the primary brake on dopaminergic activity (Barrot et al., 2012). GABAergic neurons intermingled amongst dopamine neurons in the VTA project to the NAc and LHb, as well as sending local collaterals (Brown et al., 2012; Root et al., 2014; Taylor et al., 2014). Thus, it is likely that in previous studies examining the influence of VTA GABAergic on disinhibition of dopamine neurons, that they are in fact stimulating tVTA/RMTg neurons located caudally to the VTA (van Zessen et al., 2012; Nieh et al., 2015, 2016).

Female mice show decreased qIPSC amplitude, but not frequency of quantal events from the LH GABA - tVTA/RMTg GABA projection compared to male mice, consistent with a decrease in number, function, or subunit composition of postsynaptic $\mathrm{GABA}_{\mathrm{A}}$ receptors at $\mathrm{LH}$ GABAtVTA/RMTg synapses. Notably, these effects were only observed during the asynchronous period 350 ms post optical stimulation, but not later, suggesting that this effect is specific to $\mathrm{LH}$ GABAergic inputs. LH GABA synapses were depressing at tVTA/RMTg neurons, there was a similar paired pulse ratio across all pulses. These results indicate that short term dynamics are similar at male and female tVTA/RMTg neurons with robust depression in both sexes.

Activation of LH GABAergic terminals decreases firing of tVTA/RMTg GABAergic neurons in both male and female rats. This effect was most pronounced within the first 5 pulses of the $20 \mathrm{~Hz}$ stimulation and largely driven by increased latency to fire rather than a decrease in AHP. 
Typically, a low threshold and fast inactivating $\mathrm{K}+$ current $\left(I_{\mathrm{A}}\right)$ underlies the latency to fire as it is activated during hyperpolarization and opposes membrane depolarization resulting in a delay to reach firing threshold (Kanold \& Manis, 1999). Thus, optical stimulation of the LH GABAergic input likely hyperpolarizes tVTA/RMTg neurons leading to activation of $I_{\mathrm{A}}$ and a subsequent increased latency to fire (Kanold \& Manis, 2005).

Effects of fasting on LH GABA - tVTA/RMTg GABA synapses

To examine the effects of optical stimulation of LH GABA inputs to the tVTA/RMTg, we recorded optically evoked IPSCS in ACSF where extracellular calcium was replaced with strontium which desynchronizes vesicular release to allow for the resolution of quantal synaptic events (Bekkers \& Clements, 1999). The resultant local input specific qIPSC amplitude provides a measure of unitary synaptic strength, whereas qIPSC frequency represents the number of connections or probability of release (Bekkers \& Clements, 1999; McGarry \& Carter, 2017). An acute overnight fast decreased the amplitude of LH GABA-mediated qIPSCs onto tVTA/RMTg neurons of male and female mice and decreased the frequency of qIPSCS of tVTA/RMTg neurons of female mice. This effect occurred within the first 350 ms after stimulation during the asynchronous release period. Thus, the local inhibitory drive onto tVTA/RMTg neurons after LH GABA stimulation is decreased with fasting, likely due to decreased synaptic strength and/or a possible decrease in number of local connections. Consistent with this, we observed a decrease in the optically evoked amplitude, the RRP size, and the steady-state oIPSC amplitude, of the LH GABAergic input to tVTA/RMTg GABA neurons after fasting in male and female mice that could contribute to the decrease in synaptic strength after fasting. 
The reduction of firing of tVTA/RMTg neurons induced by optical stimulation of LH GABA inputs is diminished after an acute fast. Consistent with this, approximately half the population of tVTA/RMTg neurons experienced depolarization block in response to a depolarizing step, yet in fasted mice, the number of cells with depolarization block was reduced by optical stimulation. This suggests that fasting may preserve the function of the LH GABA to tVTA/RMTg GABA input under depolarized conditions. This may be due to a fasting-induced suppression of inhibitory synaptic transmission at LH GABA - tVTA/RMTg synapses, reducing the hyperpolarizing influence from this input and thus preserving firing.

Given that fasting increases the motivation to approach and consume food (Burnett et al., 2016; Godfrey \& Borgland, 2020), it may appear counterintuitive that fasting does not change, but preserves the functional output of the LH GABA-tVTA/RMTg GABA synapses. While activation of LH GABAergic neurons produced consummatory behaviour in most (Nieh et al., 2015; Navarro et al., 2016; Marino et al., 2020), but not all paradigms (Burnett et al., 2016), its projections to the VTA likely drive behavioural activation. Supporting this, stimulation of $\mathrm{LH}_{\mathrm{GABA}^{-}}$ VTA inputs disinhibit VTA dopamine neurons and increases dopamine release in the NAC, leading to a promotion of motivated behaviours, including feeding, social interaction, generalized approach behaviours (Nieh et al., 2016) and reward predictions (Sharpe et al., 2017). Where the LH GABAergic inputs synapse within the VTA may play a role in the differential behavioural outcomes. For example, stimulation of the LH GABA input in the lateral VTA, VTA dopamine neurons, or lateral VTA GABAergic neurons ( $\mathrm{M} / \mathrm{L}$ coordinate: $1.6 \mathrm{~mm}$ ) does 
not influence feeding behaviour, and rapid feeding onset was proposed to occur through LH GABA projections to the peri-locus coeruleus area (Marino et al., 2020). However, stimulating LH GABAergic projections in the caudomedial VTA (M/L coordinate: $0.5 \mathrm{~mm}$ ) leads to rapid feeding behaviour (Nieh et al., 2015, 2016; Barbano et al., 2016). Therefore, it is possible that the differing effects of LH GABA inputs to the VTA on feeding behaviour may be due to LH inputs on different GABAergic populations with in the VTA. We recorded from caudomedial region of the VTA, a region almost exclusively GABAergic neurons, which are representative of the tVTA/RMTg population. Under sated conditions, activation of the LH GABA input suppresses firing of tVTA/RMTg GABA neurons. However, during fasting, the LH GABAergic input to VTA neurons is weakened, minimizing the hyperpolarizing effect of this input on tVTA/RMTg GABAergic neurons, such that firing of tVTA/RMTg is not further changed. Thus, the ability of LH GABA neurons to disinhibit dopamine neurons is likely preserved in fasting leading to behavioural activation. An alternative hypothesis is that suppressed firing activity of tVTA/RMTg GABA neurons during fasting could be a mechanism associated with brain energy conservation during food scarcity, as has been observed in the neocortex which allowed for the significant conservation of ATP (Padamsey et al., 2021). However, as in Padamsey et al., 2021, synaptic functioning is preserved and activation of LH GABAergic inputs to tVTA/RMTg GABAergic neurons can override this fasting-induced suppression of synaptic strength. Future studies should aim to address either of these hypotheses.

\section{Acknowledgements}

The authors would like to acknowledge the Hotchkiss Brain Institute advanced microscopy 
facility for their technical support.

\section{Funding disclosure}

This work was supported by an NSERC Discovery grant (DG-343012 / DAS-04060 to S.L.B.) and a

Canada Research Chair (950-232211). N.G. was supported by a Harley N. Hotchkiss Doctoral

Scholarship.

\section{Author Contributions:}

NG performed electrophysiological, immunohistochemical and fasting experiments. MQ

performed immunohistochemistry experiments. NG and SLB wrote first drafts of the manuscript. NG and SLB revised the manuscript.

\section{Conflict of Interest Statement:}

The authors declare no competing financial or other conflicts of interest.

\section{Data Availability}

Data is available upon request to the corresponding author. 


\section{References}

Balcita-Pedicino JJ, Omelchenko N, Bell R \& Sesack SR (2011). The inhibitory influence of the lateral habenula on midbrain dopamine cells: Ultrastructural evidence for indirect mediation via the rostromedial mesopontine tegmental nucleus. J Comp Neurol 519, 11431164.

Barbano MF, Wang HL, Morales M \& Wise RA (2016). Feeding and reward are differentially induced by activating GABAergic lateral hypothalamic projections to VTA. J Neurosci 36, 2975-2985.

Barrot M, Sesack SR, Georges F, Pistis M, Hong S \& Jhou TC (2012). Braking dopamine systems: A new GABA master structure for mesolimbic and nigrostriatal functions. J Neurosci 32, 14094-14101.

Bekkers JM \& Clements JD (1999). Quantal amplitude and quantal variance of strontiuminduced asynchronous EPSCs in rat dentate granule neurons. J Physiol 516, 227-248.

Berridge KC (2009). "Liking" and "wanting" food rewards: Brain substrates and roles in eating disorders. Physiol Behav 97, 537-550.

Betley JN, Xu S, Cao ZFH, Gong R, Magnus CJ, Yu Y \& Sternson SM (2015). Neurons for hunger and thirst transmit a negative-valence teaching signal. Nature 521, 180-185.

Bourdy R \& Barrot M (2012). A new control center for dopaminergic systems: Pulling the VTA by the tail. Trends Neurosci 35, 681-690.

Brown MTC, Tan KR, O'Connor EC, Nikonenko I, Muller D \& Lüscher C (2012). Ventral tegmental area GABA projections pause accumbal cholinergic interneurons to enhance associative 
learning. Nature 492, 452-456.

Brown PL, Palacorolla H, Brady D, Riegger K, Elmer GI \& Shepard PD (2017). Habenula-induced inhibition of midbrain dopamine neurons is diminished by lesions of the rostromedial tegmental nucleus. J Neurosci 37, 217-225.

Burnett CJ, Li C, Webber E, Tsaousidou E, Xue SY, Brüning JC \& Krashes MJ (2016). HungerDriven Motivational State Competition. Neuron 92, 187-201.

Chieng B, Azriel Y, Mohammadi S \& Christie MJ (2011). Distinct cellular properties of identified dopaminergic and GABAergic neurons in the mouse ventral tegmental area. J Physiol 589, $3775-3787$.

Cone JJ, Fortin SM, McHenry JA, Stuber GD, McCutcheon JE \& Roitman MF (2016). Physiological state gates acquisition and expression of mesolimbic reward prediction signals. Proc Natl Acad Sci U S A 113, 1943-1948.

Farrant M \& Nusser Z (2005). Variations on an inhibitory theme: Phasic and tonic activation of GABA A receptors. Nat Rev Neurosci 6, 215-229.

Fortin SM \& Roitman MF (2018). Challenges to body fluid homeostasis differentially recruit phasic dopamine signaling in a taste-selective manner. J Neurosci 38, 6841-6853.

Geddes SD, Assadzada S, Lemelin D, Sokolovski A, Bergeron R, Haj-Dahmane S \& Béique JC (2016). Target-specific modulation of the descending prefrontal cortex inputs to the dorsal raphe nucleus by cannabinoids. Proc Natl Acad Sci U S A 113, 5429-5434.

Godfrey N \& Borgland SL (2020). Sex differences in the effect of acute fasting on excitatory and inhibitory synapses onto ventral tegmental area dopamine neurons. J Physiol 598, 55235539. 
Grundy D (2015). Principles and standards for reporting animal experiments in The Journal of

Physiology and Experimental Physiology. J Physiol 593, 2547-2549.

Jacob TC, Moss SJ \& Jurd R (2008). GABAA receptor trafficking and its role in the dynamic modulation of neuronal inhibition. Nat Rev Neurosci 9, 331-343.

Jhou TC, Fields HL, Baxter MG, Saper CB \& Holland PC (2009). The Rostromedial Tegmental Nucleus (RMTg), a GABAergic Afferent to Midbrain Dopamine Neurons, Encodes Aversive Stimuli and Inhibits Motor Responses. Neuron 61, 786-800.

Kanold PO \& Manis PB (1999). Transient potassium currents regulate the discharge patterns of dorsal cochlear nucleus pyramidal cells. J Neurosci 19, 2195-2208.

Kanold PO \& Manis PB (2005). Encoding the timing of inhibitory inputs. J Neurophysio/ 93, 2887-2897.

Li H, Pullmann D, Cho JY, Eid M \& Jhou TC (2019a). Generality and opponency of rostromedial tegmental (RMTg) roles in valence processing. Elife 8, 1-19.

Li H, Pullmann D \& Jhou TC (2019b). Valence-encoding in the lateral habenula arises from the entopeduncular region. Elife 8, 1-17.

Li H, Vento PJ, Parrilla-Carrero J, Pullmann D, Chao YS, Eid M \& Jhou TC (2019c). Three Rostromedial Tegmental Afferents Drive Triply Dissociable Aspects of Punishment Learning and Aversive Valence Encoding. Neuron 104, 987-999.e4.

Lunde A \& Glover JC (2020). A versatile toolbox for semi-automatic cell-by-cell object-based colocalization analysis. Sci Rep 10, 1-26.

MacAskill AF, Cassel JM \& Carter AG (2014). Cocaine exposure reorganizes cell type- and inputspecific connectivity in the nucleus accumbens. Nat Neurosci 17, 1198-1207. 
Maguire JL, Stell BM, Rafizadeh M \& Mody I (2005). Ovarian cycle-linked changes in GABAA receptors mediating tonic inhibition alter seizure susceptibility and anxiety. Nat Neurosci 8, 797-804.

Margolis EB, Lock H, Hjelmstad GO \& Fields HL (2006). The ventral tegmental area revisited: Is there an electrophysiological marker for dopaminergic neurons? J Physiol 577, 907-924. Margolis EB, Toy B, Himmels P, Morales M \& Fields HL (2012). Identification of rat ventral tegmental area GABAergic neurons. PLoS One; DOI: 10.1371/journal.pone.0042365. Marino RAM, McDevitt RA, Gantz SC, Shen H, Pignatelli M, Xin W, Wise RA \& Bonci A (2020). Control of food approach and eating by a GABAergic projection from lateral hypothalamus to dorsal pons. Proc Natl Acad Sci U S A 117, 8611-8615.

Mazzone CM, Liang-Guallpa J, Li C, Wolcott NS, Boone MH, Southern M, Kobzar NP, Salgado IDA, Reddy DM, Sun F, Zhang Y, Li Y, Cui G \& Krashes MJ (2020). High-fat food biases hypothalamic and mesolimbic expression of consummatory drives. Nat Neurosci 23, 12531266.

McGarry LM \& Carter AG (2016). Inhibitory gating of basolateral Amygdala inputs to the prefrontal cortex. J Neurosci 36, 9391-9406.

McGarry LM \& Carter AG (2017). Prefrontal cortex drives distinct projection neurons in the basolateral amygdala. Cell Rep 21, 1426-1433.

Melis M, De Felice M, Lecca S, Fattore L \& Pistis M (2013). Sex-specific tonic 2arachidonoylglycerol signaling at inhibitory inputs onto dopamine neurons of Lister hooded rats. Front Integr Neurosci 7, 1-13.

Melón LC, Nolan ZT, Colar D, Moore EM \& Boehm SL (2017). Activation of extrasynaptic $\delta$ - 
GABAA receptors globally or within the posterior-VTA has estrous-dependent effects on consumption of alcohol and estrous-independent effects on locomotion. Horm Behav 95, $65-75$.

Nair-Roberts RG, Chatelain-Badie SD, Benson E, White-Cooper H, Bolam JP \& Ungless MA (2008). Stereological estimates of dopaminergic, GABAergic and glutamatergic neurons in the ventral tegmental area, substantia nigra and retrorubral field in the rat. Neuroscience 152, 1024-1031.

Navarro M, Olney JJ, Burnham NW, Mazzone CM, Lowery-Gionta EG, Pleil KE, Kash TL \& Thiele TE (2016). Lateral hypothalamus GABAergic neurons modulate consummatory behaviors regardless of the caloric content or biological relevance of the consumed stimuli. Neuropsychopharmacology 41, 1505-1512.

Nieh EH, Matthews GA, Allsop SA, Presbrey KN, Leppla CA, Wichmann R, Neve R, Wildes CP \& Tye KM (2015). Decoding neural circuits that control compulsive sucrose seeking. Cell 160, 528-541.

Nieh EH, Vander Weele CM, Matthews GA, Presbrey KN, Wichmann R, Leppla CA, Izadmehr EM \& Tye KM (2016). Inhibitory Input from the Lateral Hypothalamus to the Ventral Tegmental Area Disinhibits Dopamine Neurons and Promotes Behavioral Activation. Neuron 90, 1286-1298.

Padamsey Z, Katsanevaki D, Dupuy N \& Rochefort NL (2021). Neocortex saves energy by reducing coding precision during food scarcity. Neuron1-17.

Prior H, Ewart L, Bright J \& Valentin JP (2012). Refinement of the charcoal meal study by reduction of the fasting period. ATLA Altern to Lab Anim 40, 99-107. 
Root DH, Mejias-Aponte C, Zhang S, Wang H, Hoffman AF, Lupica CR \& Morales M (2014). Single rodent mesohabenular axons release glutamate and GABA. Nat Neurosci 17, 1543-1551.

Roseberry AG (2015). Acute fasting increases somatodendritic dopamine release in the ventral Tegmental area. J Neurophysiol 114, 1072-1082.

Salamone JD, Correa M, Mingote S \& Weber SM (2003). Nucleus accumbens dopamine and the regulation of effort in food-seeking behavior: Implications for studies of natural motivation, psychiatry, and drug abuse. J Pharmacol Exp Ther 305, 1-8.

Sánchez-Catalán MJ, Faivre F, Yalcin I, Muller MA, Massotte D, Majchrzak M \& Barrot M (2017). Response of the Tail of the Ventral Tegmental Area to Aversive Stimuli. Neuropsychopharmacology 42, 638-648.

Sharpe MJ, Marchant NJ, Whitaker LR, Richie CT, Zhang YJ, Campbell EJ, Koivula PP, Necarsulmer JC, Mejias-Aponte C, Morales M, Pickel J, Smith JC, Niv Y, Shaham Y, Harvey BK \& Schoenbaum G (2017). Lateral Hypothalamic GABAergic Neurons Encode Reward Predictions that Are Relayed to the Ventral Tegmental Area to Regulate Learning. Curr Biol 27, 2089-2100.e5.

Stell BM, Brickley SG, Tang CY, Farrant M \& Mody I (2003). Neuroactive steroids reduce neuronal excitability by selectively enhancing tonic inhibition mediated by $\delta$ subunitcontaining GABAA receptors. Proc Natl Acad Sci U S A 100, 14439-14444.

Stuber GD \& Wise RA (2016). Lateral hypothalamic circuits for feeding and reward. Nat Neurosci 19, 198-205.

Taylor S, Badurek S, DiLeone R, Nashmi R, Minichiello L \& Picciotto M (2014). GABAergic and Glutamatergic Efferents of the Mouse Ventral Tegmental Area. J Comp Neurol 522, 3308- 
3334.

Thanawala MS \& Regehr WG (2013). Presynaptic calcium influx controls neurotransmitter

release in part by regulating the effective size of the readily releasable pool. $J$ Neurosci 33 , $4625-4633$.

Thanawala MS \& Regehr WG (2016). Determining synaptic parameters using high-frequency activation. J Neurosci Methods 264, 136-152.

Ungless MA \& Grace AA (2012). Are you or aren't you? Challenges associated with physiologically identifying dopamine neurons. Trends Neurosci 35, 422-430.

Wise RA (2006). Role of brain dopamine in food reward and reinforcement. Philos Trans $R$ Soc $B$ Biol Sci 361, 1149-1158.

Van Zessen R, Phillips JL, Budygin EA \& Stuber GD (2012). Activation of VTA GABA Neurons Disrupts Reward Consumption. Neuron 73, 1184-1194. 


\section{Figure Legends}

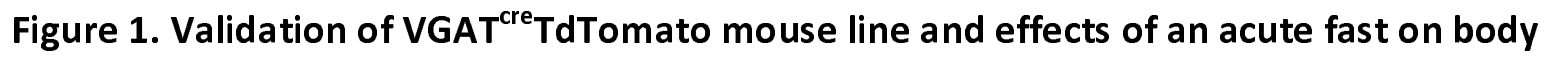
weight and consumption in male and female mice.

A, Confocal images of cells expressing VGAT ${ }^{\text {cre }} \mathrm{TdTomato}$ (red), $\mathrm{TH}$ (green), and merged in the caudal VTA. B, percent of all td-Tomato labelled cells in the VTA that are only VGAT+, and VGAT+ and TH+. Only a very small proportion of cells are both VGAT+ and TH+. C, schematic representation of time course of fast and when weights were recorded. D, mouse weight (g) prior to the $16 \mathrm{~h}$ overnight isolation or fast. Male mice weigh more than female mice. $\mathbf{E}$, chow consumed ( $\mathrm{kcal} / \mathrm{body}$ weight $(\mathrm{g})$ ) by control male and female mice during the $16 \mathrm{~h}$ overnight period of isolation. Female mice consume more food per body weight than male mice. F, body weight change between before and after the $16 \mathrm{~h}$ overnight period. Male and female fasted mice have reduced body weight after fasting. G, water consumed by male and female mice during the $16 \mathrm{~h}$ overnight period. Male and female fasted mice reduce water intake during the acute fast.

\section{Figure 2. Effects of fasting and sex on in miniature inhibitory postsynaptic currents onto} tVTA/RMTg GABA neurons of male and female mice.

A, representative examples of mIPSC recordings from male and female fasted and control mice. B, mIPSC amplitude of GABA neurons of male and female fasted and control mice. mIPSC amplitude is less in female controls than male controls. C, mIPSC frequency onto GABA neurons 
of male and female fasted and control mice. mIPSC frequency onto tVTA/RMTg neurons of female fasted and control mice was less than in male control and fasted mice.

Figure 3. Effect of sex and fasting on excitability and intrinsic membrane properties of tVTA/RMTg GABA neurons.

A, current step waveform of the 500 ms current steps. B, representative example recordings of tVTA/RMTg GABA neurons firing during a 100 pA step. C, frequency-current plot demonstrating a decrease in excitability of tVTA/RMTg GABA neurons following fasting in male and female mice. D, input resistance (mOhms) of tVTA/RMTg neurons of male and female fasted and control mice. No change in males or females following fasting. E, resting membrane potential (mV) of male and female fasted and control mice. tVTA/RMTg GABA neurons are more depolarized following fasting in both males and females.

Figure 4. Validation of ChR2 in LH GABA neurons and their projection to tVTA/RMTg GABA neurons.

A, slide scanner and confocal images of cells expressing VGAT ${ }^{\text {cre }}$ TdTomato (red), DIO-ChR2 (green), and merged. B, representative example recordings of optically stimulated action potentials in LH GABA neurons at $5 \mathrm{~Hz}, 10 \mathrm{~Hz}$, and $20 \mathrm{~Hz}$. C, representative example recordings of optically stimulated currents in tVTA/RMTg GABA neurons at $5 \mathrm{~Hz}, 10 \mathrm{~Hz}$, and $20 \mathrm{~Hz}$. D, representative example recording of the change in membrane potential during a $1 \mathrm{~s}$ optical stimulation of a LH GABA neuron. E, representative example recording of an optically stimulated $1 \mathrm{~s}$ current in a tVTA/RMTg GABA neuron. $\mathbf{F}$, schematic of a sagittal brain slice, 
demonstrating the location of the LH injection and recording of tVTA/RMTg GABA neurons. G, optically evoked currents in tVTA/RMTg GABA neurons are blocked with bath application of picrotoxin. $\mathbf{H}$, optically evoked currents of tVTA/RMTg GABA neurons are blocked with bath application of TTX, and then restored following bath application of TTX and 4-AP.

\section{Figure 5. Strontium-ind uced asynchronous quantal IPSCs at LH GABA to tVTA/RMTg GABA}

\section{synapses.}

A, representative example recordings of optically induced asynchronous qISPCS at LH GABA to VTA GABA synapse of male and female fasted and control mice. B, optically evoked qIPSC amplitude (pA) at LH GABA to tVTA/RMTg GABA synapses from 50 to 350 ms following optical stimulation. The amplitude of qIPSCs decreased following fasting in both male and female mice. The qIPSC amplitude was also smaller in females compared to males in both fasted and control mice. C, optically evoked qIPSC amplitude (pA) at LH GABA to tVTA/RMTg GABA synapses from 700 to 1000 ms following optical stimulation. Fasting does not affect the qIPSC amplitude during this time period. D, qIPSC frequency $(\mathrm{Hz})$ at LH GABA to tVTA/RMTg GABA synapses from 50 to 350 ms following optical stimulation. The frequency of qIPSCs decreased following fasting in female mice. E, qIPSC frequency $(\mathrm{Hz})$ at LH GABA to tVTA/RMTg GABA synapses from 700 to 1000 ms following optical stimulation. Fasting does not affect the qIPSC amplitude during this time period.

Figure 6. Short-term synaptic plasticity at tVTA/RMTg GABA synapses after an optically induced $20 \mathrm{~Hz}$ train stimulation of LH GABAergic terminals. 
A, representative example recordings of optically induced currents at $20 \mathrm{~Hz}$ at LH GABA to tVTA/RMTg GABA synapses of male and female fasted and control mice. B, time course showing the average oIPSC amplitude for all 20 pulses in male fasted and control mice. $\mathbf{C}$, time course showing the average oIPSC amplitude for all 20 pulses in female fasted and control mice. D, olPSC amplitude (pA) of the first pulse in the $20 \mathrm{~Hz}$ stimulation. oIPSC amplitude decreases following fasting in both male and female mice. E, representative example of the cumulative amplitude, used to calculate the readily releasable pool ( $\left.\mathrm{RRP}_{\text {train, }} \mathrm{pA}\right)$ and the steady state olPSC amplitude $(\mathrm{pA})$, from recordings of a male and female fasted and control tVTA/RMTg GABA

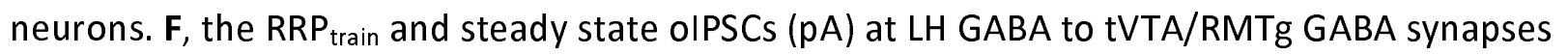
in male and female control and fasted mice. The $\mathrm{RRP}_{\text {train }}$ decreases following fasting in both male and female mice. $\mathbf{G}$, the steady-state oIPSC amplitude at LH GABA to tVTA/RMTg GABA synapses in male and female control and fasted mice. The steady-state oIPSC amplitude decreases following fasting in both male and female mice. $\mathbf{H}$, the mean eYFP fluorescence intensity in male and female control and fasted mice. The eYFP fluorescence intensity is not different between control or fasted male or female mice. I, Pn/P1 plotted for the first 5 pulses of the optically induced $20 \mathrm{~Hz}$ train stimulation in male control and fasted mice. A paired pulse depression was observed in both male control and fasted mice. J, Pn/P1 plotted for the first 5 pulses of the optically induced $20 \mathrm{~Hz}$ train stimulation in female control and fasted mice. A paired pulse depression was observed in both female control and fasted, but there was less of a depression following fasting. 


\section{Figure 7. Effects of optical stimulation of the LH GABA input on the firing of tVTA/RMTg GABA} neurons.

A, representative example recordings of action potentials from tVTA/RMTg GABA neurons induced through a 1 s, 100 pA current step, before (black) and with (blue) an optically induced $20 \mathrm{~Hz}$ stimulation of LH GABA terminals in male and female fasted and control mice. B, a time course over the $1 \mathrm{~s}$ current step of the average number of action potentials in each $100 \mathrm{~ms}$ bin in male control and fasted before the optical stimulation. $\mathbf{C}$, a time course over the $1 \mathrm{~s}$ current step of the average number of action potentials in each $100 \mathrm{~ms}$ bin in male control and fasted with the optical stimulation. D, average number of action potentials during the $1 \mathrm{~s}$ current step in male mice before and with optical stimulation of LH GABA terminals in the tVTA/RMTg. Optical stimulation of the LH GABA neuron terminals in the tVTA/RMTg did not affect the average number of action potentials during the complete $1 \mathrm{~s}$ current step. $\mathbf{E}$, average number of action potentials during the first $200 \mathrm{~ms}$ of the current step in male mice before and during optical stimulation. The average number of action potentials during the first $200 \mathrm{~ms}$ of the current step decreased with optical stimulation of LH GABA neuron terminals in the tVTA/RMTg. F, a time course over the 1 s current step of the average number of action potentials in each $100 \mathrm{~ms}$ bin in female control and fasted before the optical stimulation. G, a time course over the $1 \mathrm{~s}$ current step of the average number of action potentials in each $100 \mathrm{~ms}$ bin in female control and fasted during optical stimulation. $\mathbf{H}$, average number of action potentials during the $1 \mathrm{~s}$ current step in female mice before and during optical stimulation. The average number of action potentials in the $1 \mathrm{~s}$ current step decreased with the optical stimulation. I, average number of action potentials during the first $200 \mathrm{~ms}$ of the current step in 
female mice before and during optical stimulation. The average number of action potentials in the first $200 \mathrm{~ms}$ of the current step decreases with optical stimulation. J, the change in AP number (\%) during the first 200 ms of the current step induced by the optical stimulation in male and female control and fasted mice. The change in AP number (\%) is decreased following fasting in both males and female mice. $\mathbf{K}$, The proportion of cells with depolarization block during the 1s $100 \mathrm{pA}$ current step in control and fasted, and pre-OPTO and OPTO. Following fasting, the number of cells with depolarization block decreased when the $1 \mathrm{~s}$ current step was paired with the optical stimulation.

Figure 8. Effects of optical stimulation of the LH GABA input on action potential properties of tVTA/RMTg GABA neurons.

A, latency to fire (ms) an action potential during the $1 \mathrm{~s}$ current step before and with optical stimulation of the LH input in male mice. The latency to fire was greater with optical stimulation in male mice. B, latency to fire (ms) an action potential during the $1 \mathrm{~s}$ current step before and with optical stimulation in female mice. The latency to fire was greater with the optical stimulation in female mice. C, The change in latency to fire (\%) from LH GABA optical stimulation in male and female, fasted and control mice. Following fasting, the change in latency to fire (\%) induced by optical stimulation of LH GABA terminals decreased. D, a time course over the $1 \mathrm{~s}$ current step of the average AHP $(\mathrm{mV})$ in each $100 \mathrm{~ms}$ bin in male control and fasted before optical stimulation. E, a time course over the $1 \mathrm{~s}$ current step of the average AHP (mV) in each $100 \mathrm{~ms}$ bin in male control and fasted with the optical stimulation. $\mathbf{F}$, the average AHP (mV) during the $1 \mathrm{~s}$ current step in male control and fasted, pre-OPTO and OPTO. 
The average AHP (mV) during the 1 s current step was not affected by optical stimulation in male mice. G, the average AHP (mV) during the first $200 \mathrm{~ms}$ of the current step in male control and fasted, pre-OPTO and OPTO. The average AHP during the first $200 \mathrm{~ms}$ of the current step was not affected by optical stimulation in male mice. $\mathbf{H}$, a time course over the $1 \mathrm{~s}$ current step of the average AHP in each $100 \mathrm{~ms}$ bin in female control and fasted mice before optical stimulation. I, a time course over the $1 \mathrm{~s}$ current step of the average AHP in each $100 \mathrm{~ms}$ bin in female control and fasted mice with optical stimulation. J, the average AHP during $1 \mathrm{~s}$ current step in female control and fasted, pre-OPTO and OPTO. The average AHP during the $1 \mathrm{~s}$ current step was not affect by optical stimulation in female mice. $\mathbf{K}$, the average AHP during the first $200 \mathrm{~ms}$ of the current step in female control and fasted, pre-OPTO and OPTO. The average AHP during the first $200 \mathrm{~ms}$ of the current step was not affected by optical stimulation in female mice.

Figure 9. Effects of optical stimulation of the LH GABA input on the action potential height of tVTA/RMTg GABA neurons.

A, a time course over the $1 \mathrm{~s}$ current step of the average AP height ( $\mathrm{mV}$ ) in each $100 \mathrm{~ms}$ bin in male control and fasted before optical stimulation. B, a time course over the $1 \mathrm{~s}$ current step of the average AP height in each $100 \mathrm{~ms}$ bin in male control and fasted with optical stimulation. C, the average AP height during the $1 \mathrm{~s}$ current step in male control and fasted, pre-OPTO and OPTO. The average AP height during the $1 \mathrm{~s}$ current step was not affected by optical stimulation in male mice. $\mathbf{D}$, the average AP height during the first $200 \mathrm{~ms}$ of the current step in male control and fasted, pre-OPTO and OPTO. The average AP height during the first $200 \mathrm{~ms}$ of the 
current step increased with optical stimulation of LH GABA terminals in the VTA in male mice. E, a time course over the $1 \mathrm{~s}$ current step of the average AP height in each $100 \mathrm{~ms}$ bin in female control and fasted before optical stimulation. F, a time course over the 1 s current step of the average AP height $(\mathrm{mV})$ in each $100 \mathrm{~ms}$ bin in female control and fasted during optical stimulation. G, the average AP height during the $1 \mathrm{~s}$ current step in female control and fasted, pre-OPTO and OPTO. The average AP height during the $1 \mathrm{~s}$ current step was not affected by optical stimulation in female mice. $\mathbf{H}$, the average AP height during the first $200 \mathrm{~ms}$ of the current step in female control and fasted, pre-OPTO and OPTO. The average AP height during the first 200 ms of the current step increased with optical stimulation of LH GABA terminals in the tVTA/RMTg of female mice. 
Figure 1.

A.

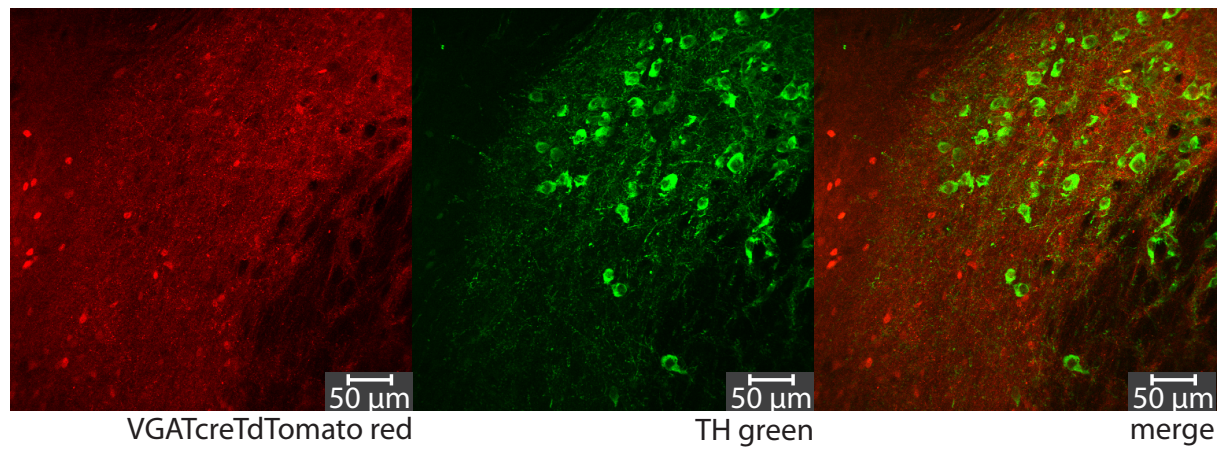

B.

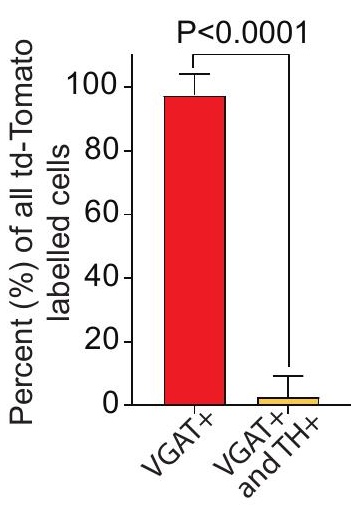

C.

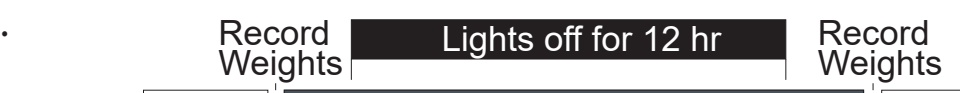

$\begin{array}{ll}\text { Unfasted } & \text { Isolate } \\ \text { Mice }\end{array}$

Lights off for $12 \mathrm{hr}$

Weights

Fasted

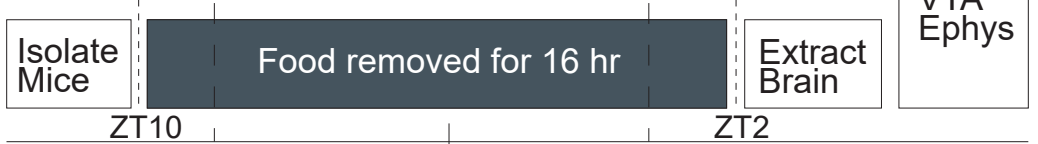

Day 1

Day 2

D.

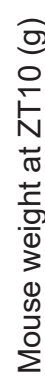

E.

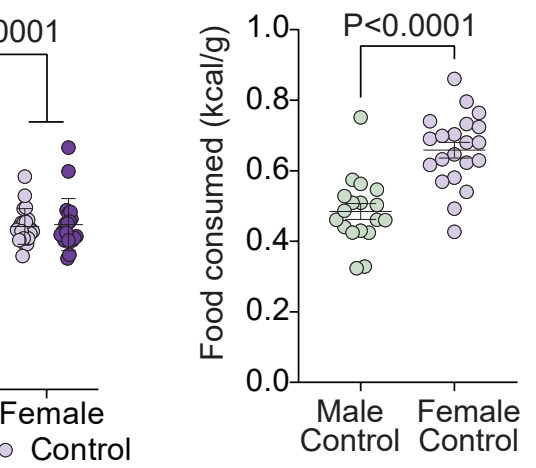

F.

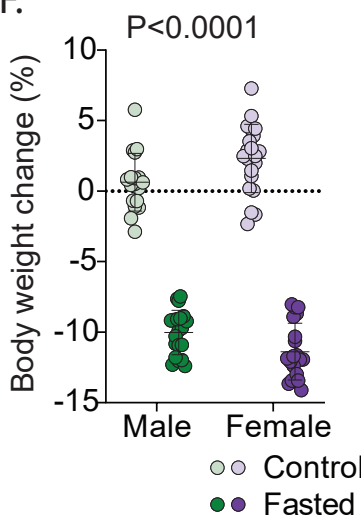

G.

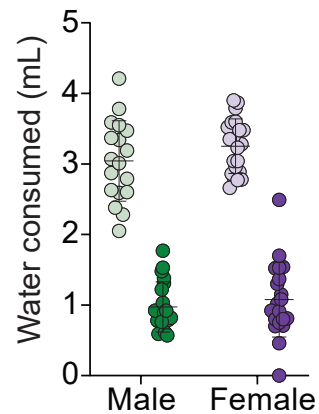

○ Control

- Fasted

- . Fasted $\mathrm{P}<0.0001$

○ Control

— Fasted $P<0.0001$ 
Figure 2.

A.

Male Control

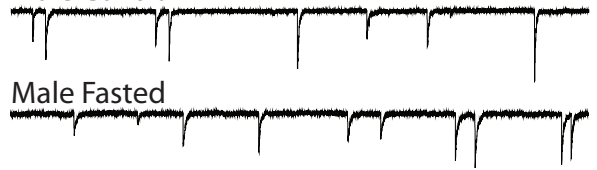

Female Control

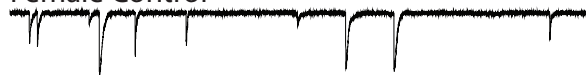

Female Fasted

B.

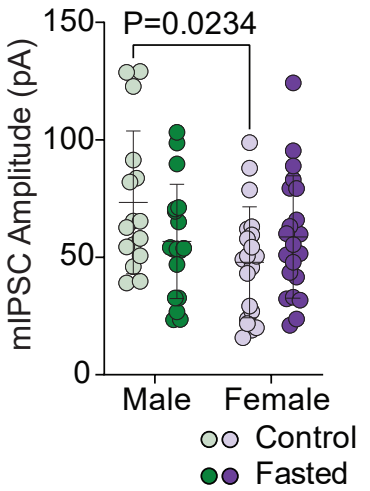

C.

$$
\text { C. } \quad \mathrm{P}=0.0245
$$

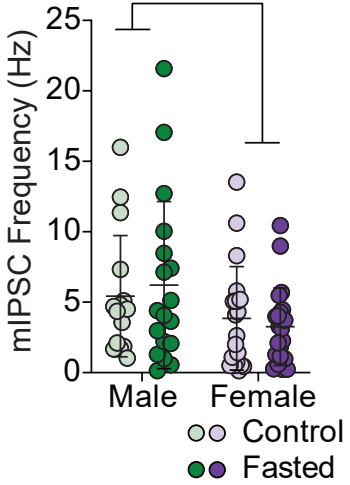


Figure 3.

A.

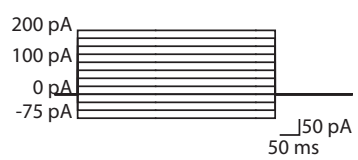

B.<smiles>C=CC(=C)CC(C)CCCC</smiles><smiles>C#CCCCCC</smiles>

Female Fasted

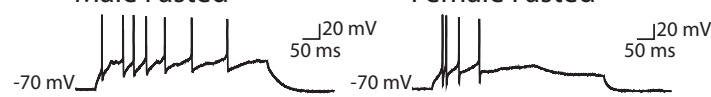

C.

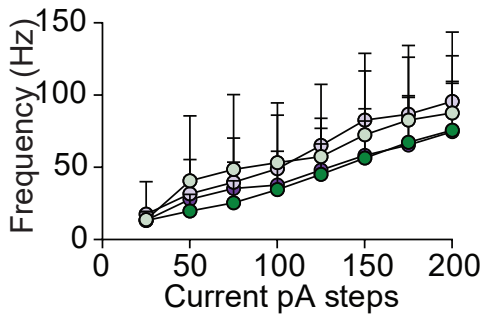

- Male Control $\mathrm{P}=0.02$

- Female Control

- Female Fasted $\mathrm{P}=0.03$

D.

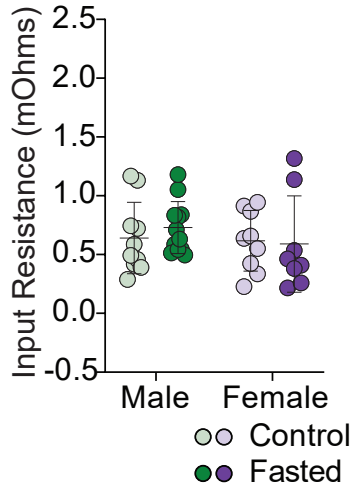

E.

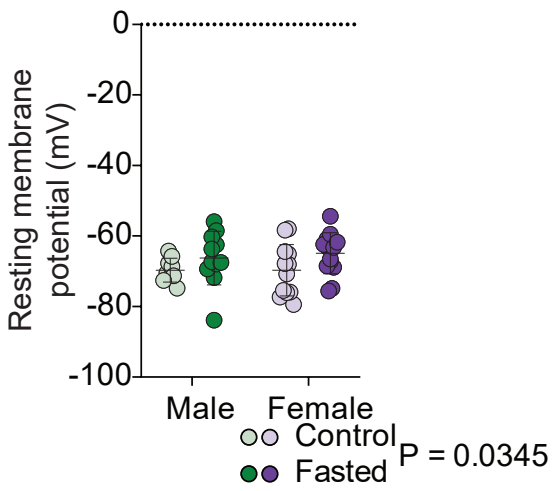


Figure 4.

A.

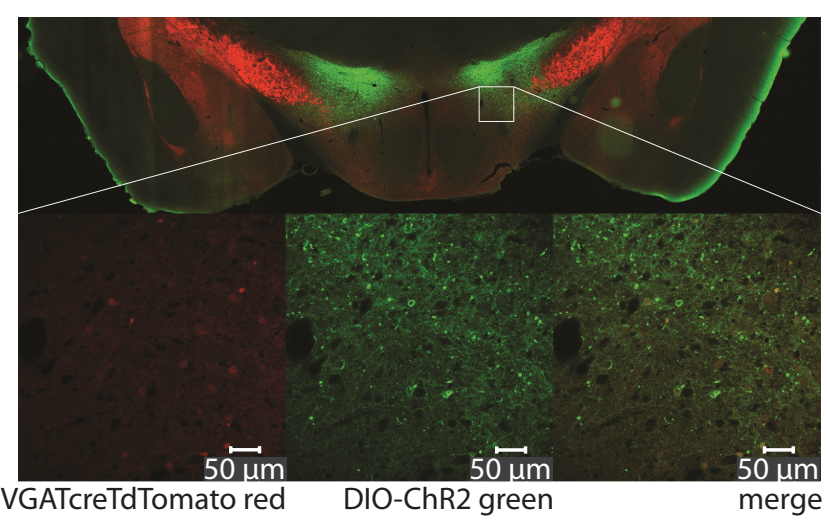

B.

LH - Current Clamp at -70mV

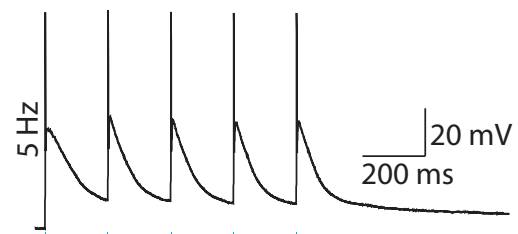

C. VTA - Voltage Clamp at - $70 \mathrm{mV}$

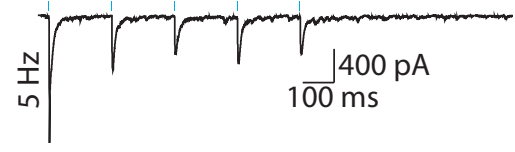

LH - Current Clamp at -70mV

D.

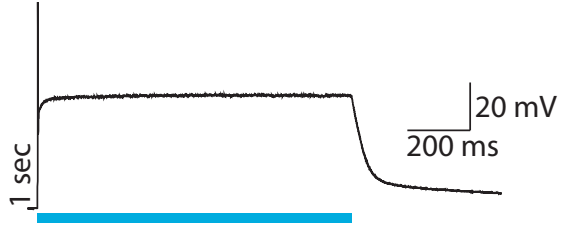

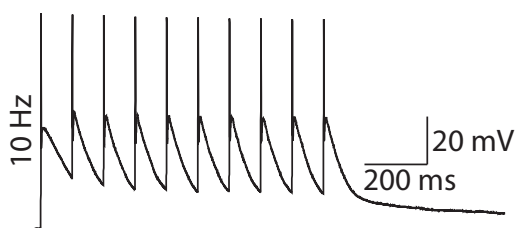

VTA - Voltage Clamp at - 70mV

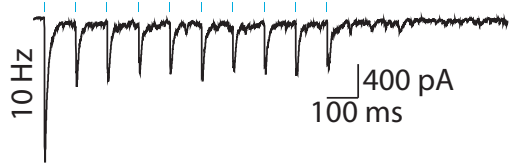

E.
LH - Current Clamp at -70mV

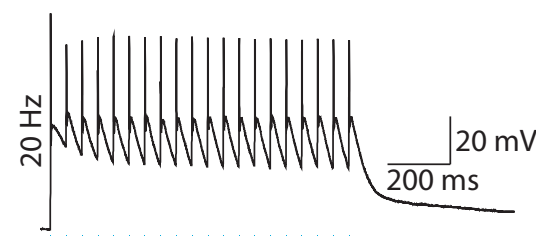

VTA - Voltage Clamp at - 70mV

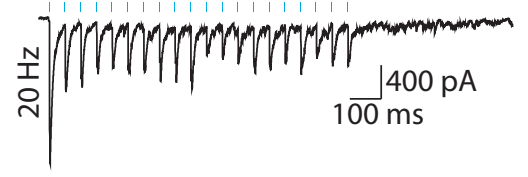

F.

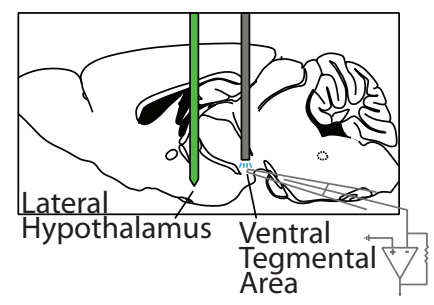

G. $\quad P=0.0114$
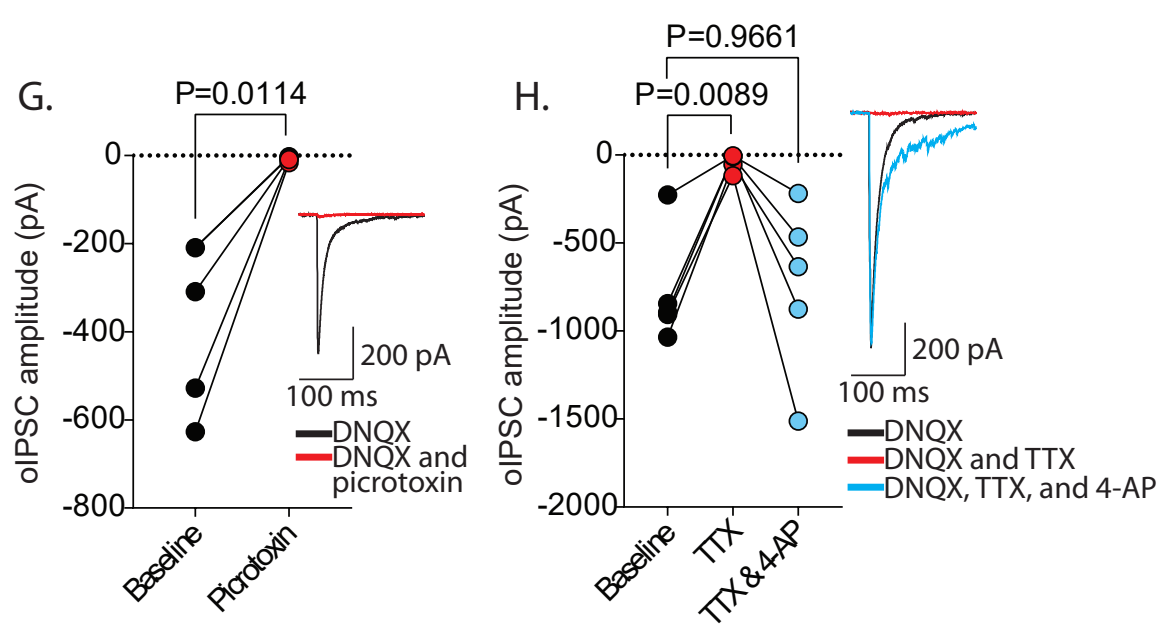
Figure 6.

A.

Male Control

ma $500 \mathrm{~ms}$

Male Fasted

$500 \mathrm{~ms}$
$200 \mathrm{pA}$

Female Control

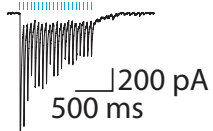

Female Fasted $500 \mathrm{~ms}$
B.

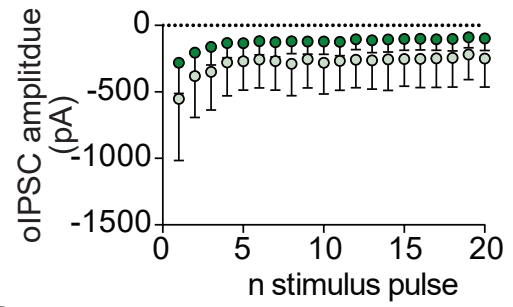

C.

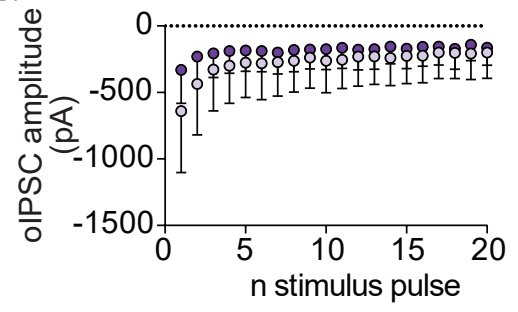

- Male Control - Male Fasted

- Female Control - Female Fasted

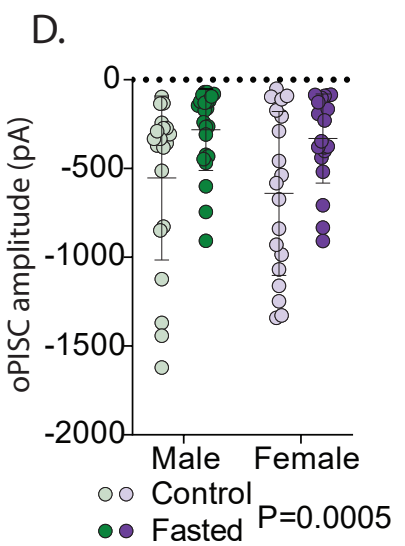

E.
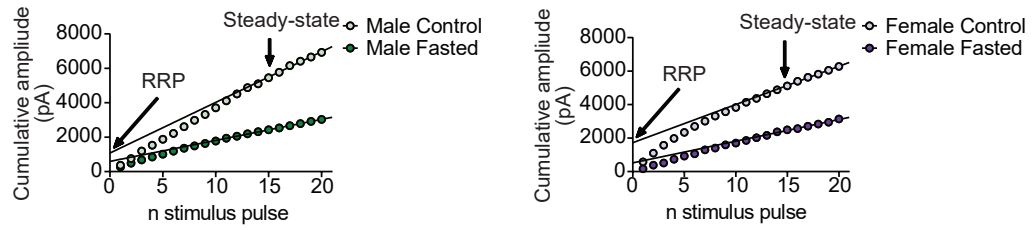

F.

G.
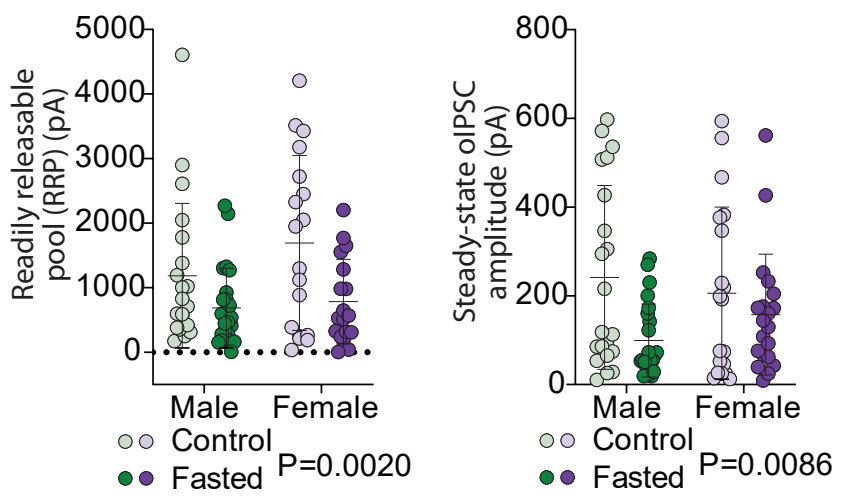

H.

I.

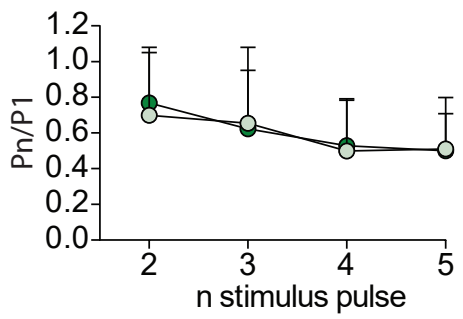

- Male Control
- Male Fasted
J.

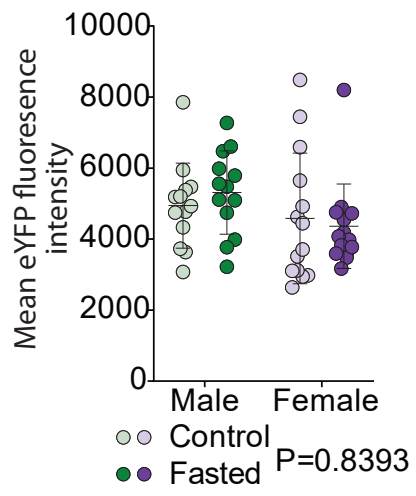

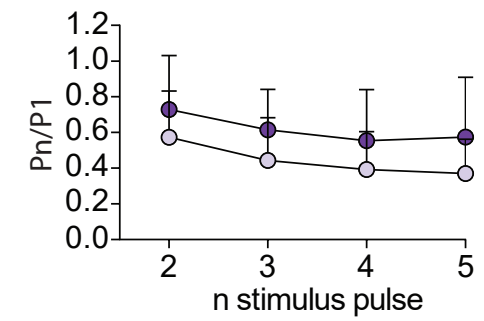

O Female Control
- Female Fasted 
A. Figure 7.

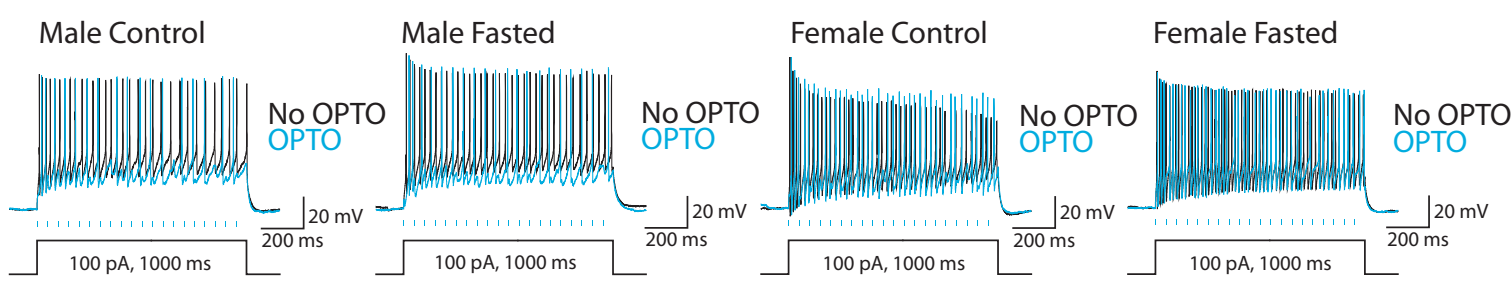

B.

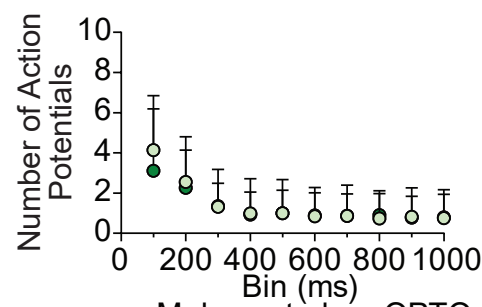

- Male control preOPTO

C. ᄃ $10^{\circ}$ Male fasted preOPTO

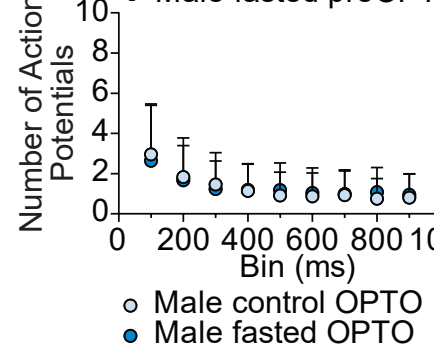

D.

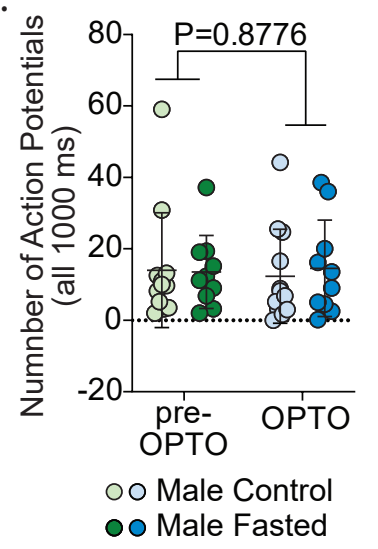

H.

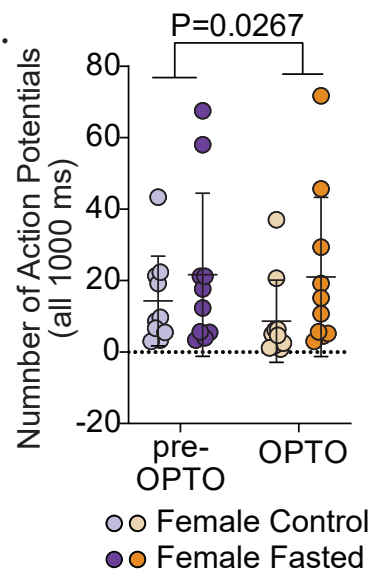

E.

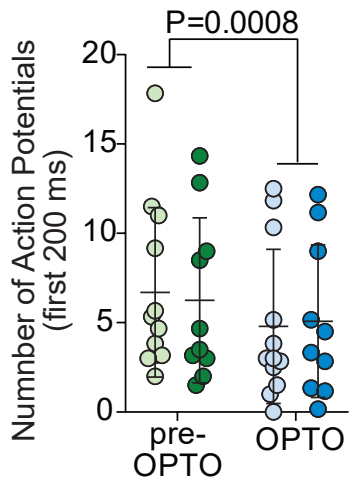

o o Male Control

o Male Fasted

F.

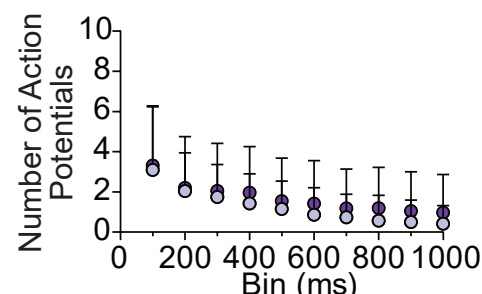

- Female control preOPTO

G.
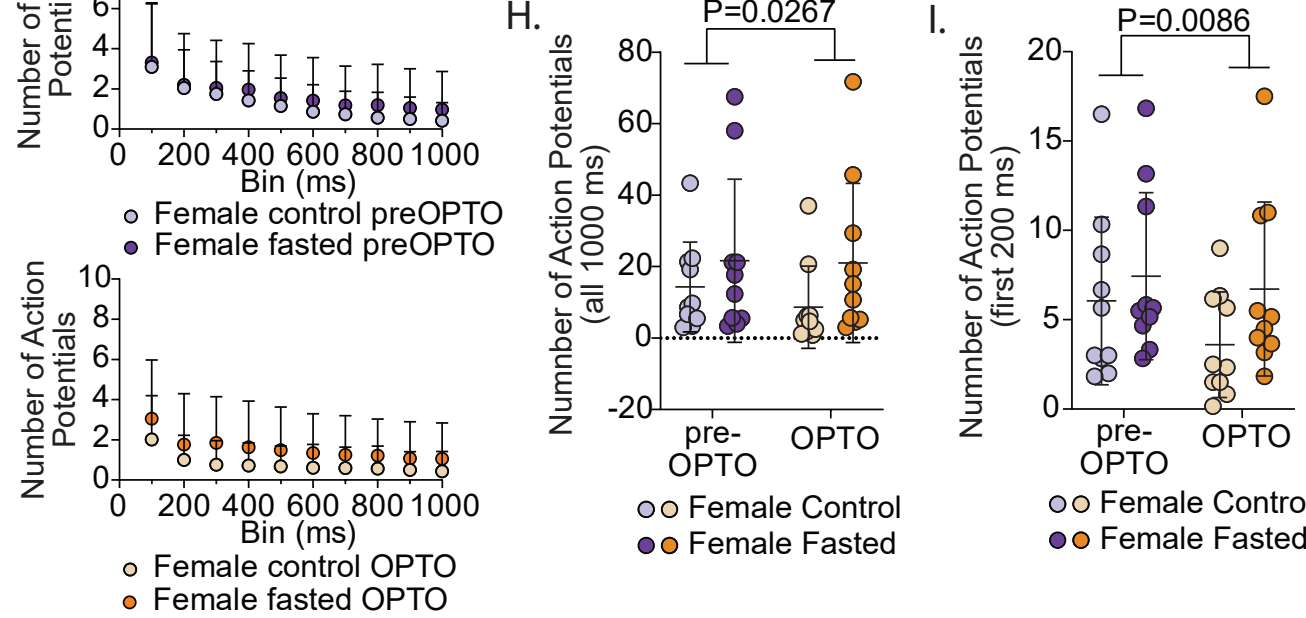

o o Female Control

- o Female Fasted

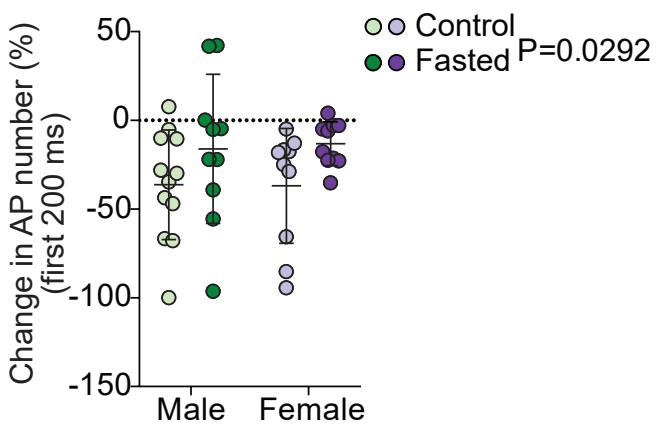

K.

PreOPTO

Control

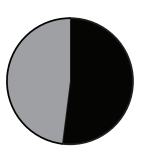

$\mathrm{P}=0.2380$

OPTO
Fasted

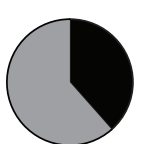

$\square$ No Depol Block 
Figure 8.

A.

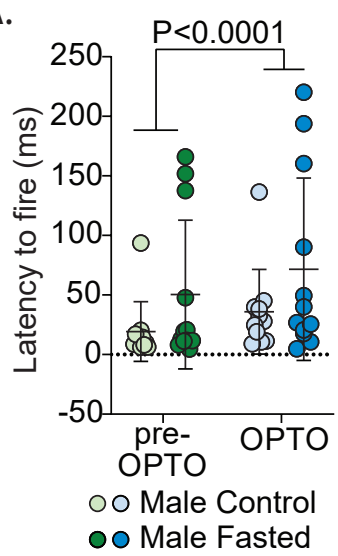

B.

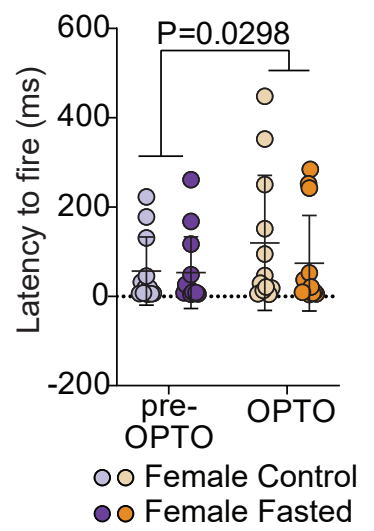

C.

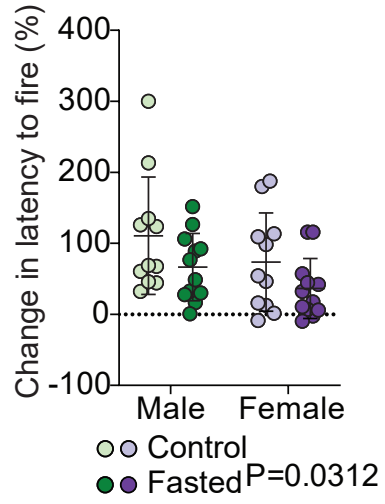

D.

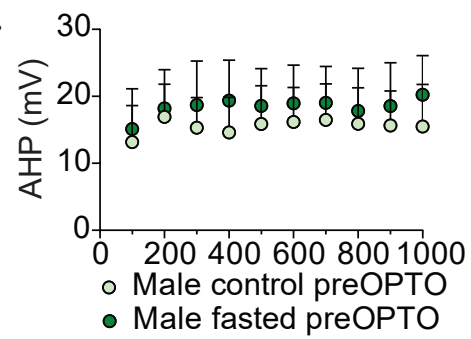

E.
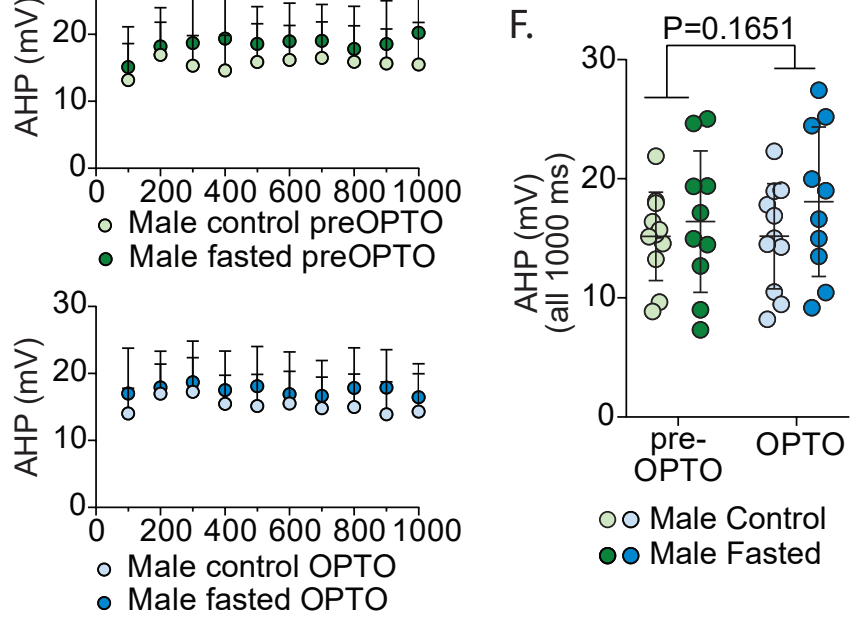

o o Male Control

○o Male Fasted

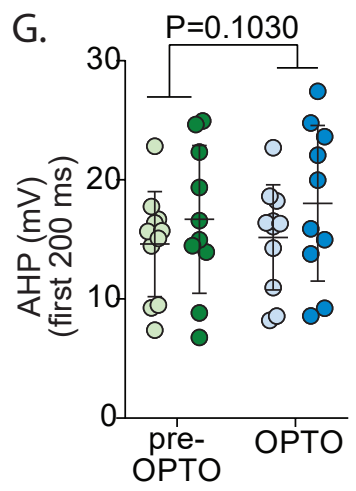

o o Male Control oo Male Fasted

$\mathrm{H}$.

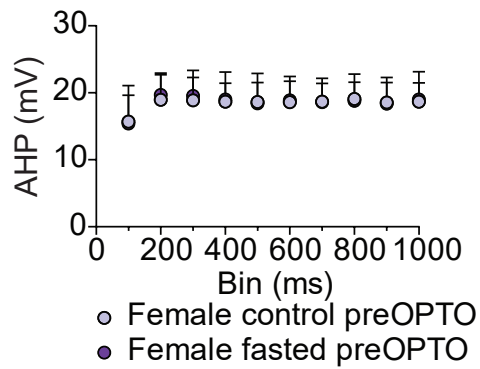

I.

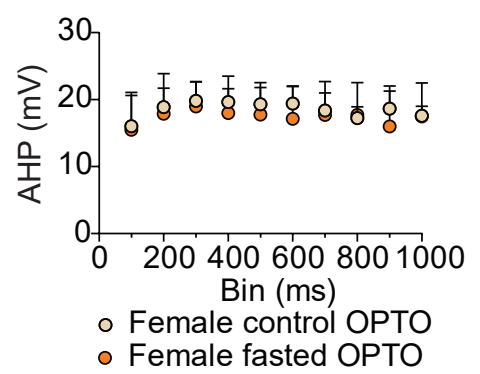

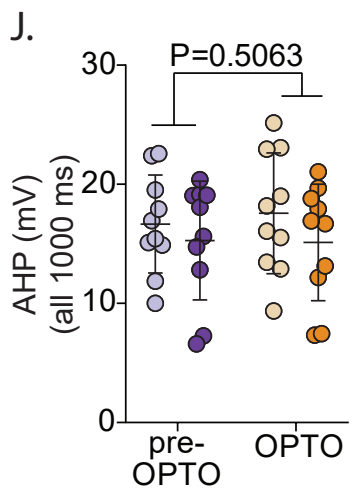

o o Female Control - o Female Fasted

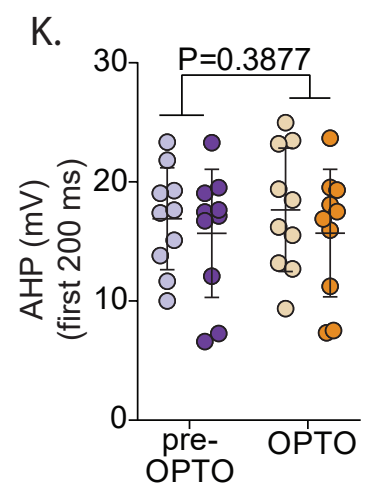

o o Female Control ○o Female Fasted 
Figure 9.

A.

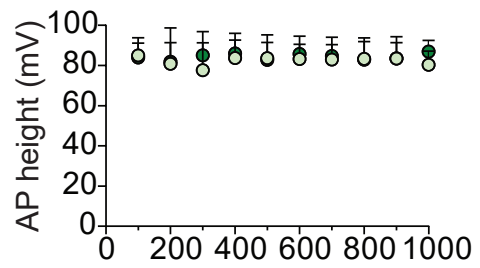
Bin (ms)

- Male control preOPTO

B.

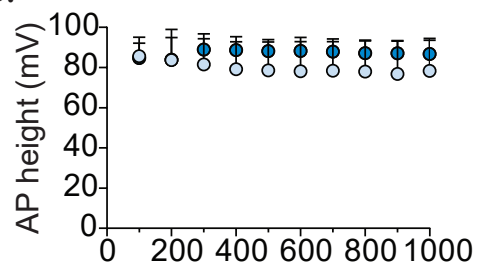
Bin (ms)

- Male control OPTO

- Male fasted OPTO

E.

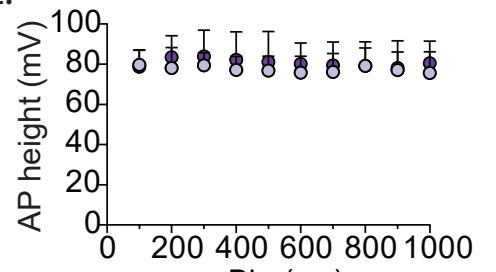
Bin (ms)

- Female control preOPTO

F.

- Female fasted preOPTO

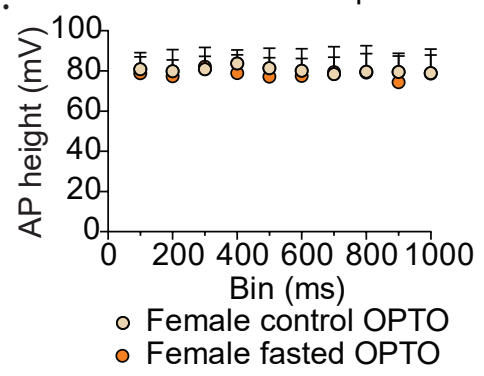

C.

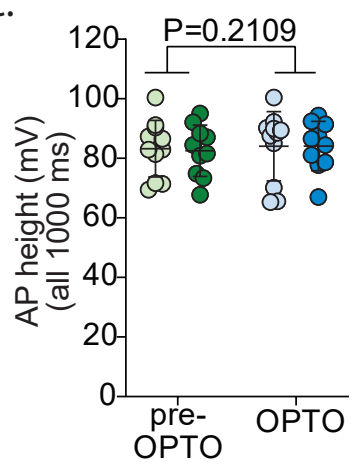

o o Male Control

○o Male Fasted

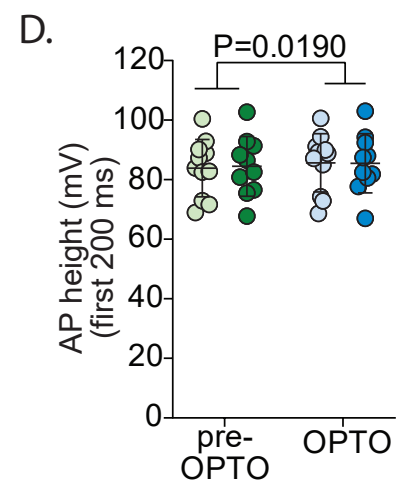

o o Male Control

oo Male Fasted
G.

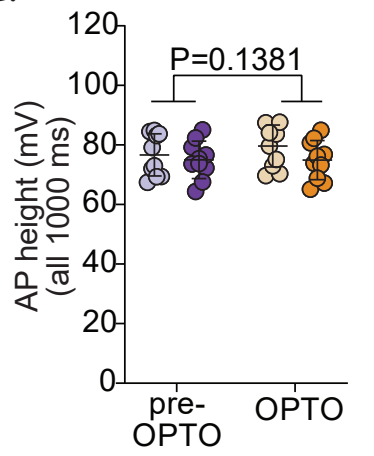

o o Female Control ○o Female Fasted
H.

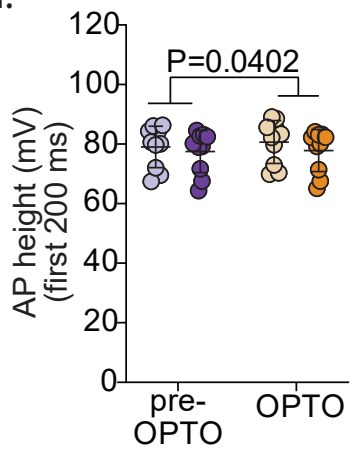

oo Female Control - o Female Fasted 